\title{
Computationally efficient reduction of modal data from finite element models by nested sets of B-splines
}

\author{
Diego Cárdenas ${ }^{1}$, Hugo Elizalde ${ }^{1, *}$, Oliver Probst ${ }^{2}$, Walter Lacarbonara ${ }^{4}$, Pier Marzocca ${ }^{3}$, Enrique \\ Aguilar $^{1}$, Ricardo Ramirez ${ }^{2}$ \\ ${ }^{1}$ Tecnológico de Monterrey, School of Engineering and Science, Calle del Puente 222 Col. Ejidos de \\ Huipulco Tlalpan, CP14380, México, D.F. \\ ${ }^{2}$ Tecnológico de Monterrey, School of Engineering and Science, Av. Eugenio Garza Sada 2015 Sur, Col. \\ Tecnológico, Monterrey, México CP64849. \\ ${ }^{3}$ RMIT University, Aerospace, Mech. \& Manuf. Eng. Department, PO Box 71, Bundoora, Victoria 3083, \\ Australia. \\ ${ }^{4}$ Dipartimento di Ingegneria Strutturale, Universitá degli Studi di Roma La Sapienza, Via Eudossiana 18, \\ 00184 Rome, Italy \\ *Corresponding author: hugo.elizalde@itesm.mx
}

\begin{abstract}
This paper introduces a novel methodology for the reduction of modal data from complex finite element (FE) models, resulting in a highly accurate and computationally efficient model termed B-SAFE (B-splinebased Semi-Analytical Finite Element model). It is conceptually similar to a method based on nested sets of polynomials proposed earlier by the authors (Poly-SAFE) but provides greater accuracy in the case of complex structures and greater ease of use, limiting the need for user interventions and facilitating automated processing. B-SAFE is constructed via the reduction of a standard displacement-based FE model down to a semi-analytical function built from nested B-splines recursively fitted to modal data, which eliminates the need of high-order polynomials and heavy user-intervention required by Poly-SAFE for the case of complex modal patterns. The present work describes the non-trivial modifications to the original methodology, and shows how fewer and more efficient semi-analytical functions representing field variables such as displacement, strain and stress can be obtained. The new method is exemplified through a numerical case study of a $9.2 \mathrm{~m}$ composite wind turbine blade, showing that improved numerical stability and robustness as well as a systematic handling of general geometries can be obtained without user intervention.
\end{abstract}

Keywords: Thin-Walled Composite Structure; Finite Element Model; Modal Reduction; Semi-Analytical; B-splines 


\section{Introduction}

The aeroelastic analysis of flexible structures represents enormous theoretical and computational challenges related to the iterative solution of sizeable, coupled and possibly nonlinear models, in many cases still untreatable with current computational capabilities [1-3]. In view of this, the evaluation of the stress field and the application of failure criteria for layered materials are generally done with simplified models, thus restricting their applicability and adding uncertainty to any design optimization attempt [47]. Historically, due to the high cost and scarcity of computing power, the development of reducedorder structural models attracted wide interest [8-12]. Today, despite the exponential growth and decreasing cost of computational resources, the increasingly complex endeavours pursued by researchers and engineers make reduced-order models equally relevant [13-18].

In a recent publication [19], the authors introduced the Poly-SAFE ("Polynomial Semi Analytical Finite Element") method, a reduced-order structural model designed to condense the modal data output of a full-size finite element (FE) model into load-independent semi-analytical functionals via nested polynomials recursively fitted to the modal data. It was shown [19] that Poly-SAFE is capable of retrieving the displacement field at any model coordinate, not restricted to FE nodes, upon simple evaluation of the functional, with an accuracy comparable to that of its parent FE model. Although capable of handling general geometries, the method is particularly suitable for the analysis of Thin-

Walled Composite Structures (TWCS) such as those found in large wind turbine blades, slender wings and antennas, among other examples [13-18]. Nonetheless, the use of canonical polynomials (e.g. based on finite power series) in complex modal patterns required either very high-order fits, making them prone to both fitting and numerical instabilities, or a heavy user-involvement to partition the data into piecewise-smooth portions which were fitted individually by low-order polynomials.

This paper introduces a major improvement to Poly-SAFE for alleviating the aforementioned issues based on the usage of B-splines [20,21], motivating the naming of the new method as B-SAFE ("B-splinebased Semi Analytical Finite Element"). The novel approach allows a similar but more robust representation of semi-analytical functionals as well as an extension of the capabilities of the original method due to the advanced arithmetic/differential manipulation techniques currently available for Bsplines [22]. Moreover, complex modal patterns can be tackled in a straightforward way without any user-involvement thanks to the local support feature of B-splines, allowing for low-order global fits while still capturing enough detail for data sets with high curvature [23]. While the latter argument is well known and true for most kind of data, it implies non-trivial modifications to the original methodology, the description of which is one of the main purposes of this paper. The rest of the document is organized as follows: Section 2 briefly reviews the fundamentals of B-splines while Section 3 provides a summarized step-by-step account of the novel method introduced in this paper (B-SAFE), also illustrated by results obtained with a representative thin-walled test structure. A validation of the new approach for the case of a $9.2 \mathrm{~m}$ composite wind turbine blade, including a comparative of Poly-SAFE versus BSAFE, is presented in Section 4. Concluding remarks with an outlook on future work are provided in Section 5. 


\section{Brief Fundamentals of B-splines}

A canonical B-spline curve is defined by a set of $n$ parametric functions $\boldsymbol{B}(t): t \rightarrow \mathfrak{R}^{n}$ which can be viewed as polynomial segments of the same order (degree plus one) $\kappa$ joined at breakpoints (so-called knots) and having $C^{e}$ continuity conditions [21]. In contrast to power series, the magnitude of the Bspline's fitting error can be reduced by increasing the number of polynomial segments while maintaining the same global order of the overall function [20], allowing datasets with high-curvature regions to be accurately reproduced by low-order (i.e. $3^{\text {rd }}$ ) polynomial segments. A B-spline curve defined in a Cartesian coordinate system $\varsigma, \phi$ can be written as:

$$
\boldsymbol{B}(t)=\sum_{a=1}^{N_{Q}} \psi_{a, \kappa}(t) \boldsymbol{Q}_{a}(\varsigma, \phi)
$$

where the basis polynomial functions $\psi_{a, k}: t \rightarrow[0,1], a=1 \ldots N_{Q}$, have a common order $\kappa$ and $\boldsymbol{Q}_{a}(\varsigma, \phi)$ are a set of $N_{Q}$ coefficients or control points [21]. A generic B-spline $(n=2)$ is shown in Figure 1, where the external hull (a.k.a control polygon) is constructed by joining the control points, the latter providing the means for locally controlling the B-spline's shape. The knot-vector $T=\left\{t_{0}, t_{1}, \ldots, t_{l}\right\}$, gathering $l+1$ knots, each a scalar value representing a particular arc-length position along the B-spline path, is used by the Mansfield-De Boor-Cox's recursion algorithm [21, 26] for an efficient computation of the basis functions $\psi_{a, \kappa}$. The number of knots, number of control points and the order of the B-spline cannot be set independently but are tied by the equality $N_{Q}=l-\kappa+1$.

While B-splines are often used to exactly reproduce and interpolate a set of data points, another important application is curve fitting. In a curve fitting context, an ordered set of data points $\boldsymbol{\tau}_{j}$ $(j=0 \ldots p$, where $p>l+1)$ is modelled as

$$
\tau_{j, \phi}=f\left(\tau_{j, \varsigma}\right)+\varepsilon, \text { for } j=0,1, \ldots, p
$$

where $\tau_{j, \varsigma}, \tau_{j, \phi}$ stand for the $\zeta, \phi$ coordinates of point $\tau_{j}, \varepsilon$ represents a noise or error function and $f$ is a smoothing function $f:\left[\tau_{0, \varsigma}, \tau_{p, \phi}\right] \rightarrow \mathfrak{R}$. In the present case the latter has the form of a single B-spline function $B$, so here $n$ is fixed to 1 , with $t=\left[\tau_{0, \varsigma}, \tau_{p, \phi}\right]$. As a consequence, knots have a value in $\varsigma$ while the coefficients $\boldsymbol{Q}_{a}(\varsigma, \phi)$ have a value in $\phi$. Once that all knots have been assigned, the $N_{Q}$ basis functions $\psi_{a, \kappa}$ are built recursively for the predefined order $\kappa$, and the $N_{Q}$ control points $\boldsymbol{Q}_{a}(\varsigma, \phi)$ are found by minimizing an error function between the points $\boldsymbol{\tau}_{j}$ and corresponding points generated by the B-spline: 


$$
\frac{\partial}{\partial Q_{a}}\left[\sum_{j=0}^{p}\left(B\left(\tau_{j, \zeta}\right)-\tau_{j, \phi}\right)^{2}\right]=0, \text { for } a=1,2 \ldots N_{Q}
$$

Fit goodness can be measured by the coefficient of determination [27]:

$$
R^{2} \equiv 1-\frac{S S_{r e s}}{S S_{\text {tot }}}
$$

where $\mathrm{SS}_{\text {res }}$ and $\mathrm{SS}_{\text {tot }}$ are the residual sum of squares and the total sum of squares, respectively.

\section{The B-SAFE model}

As mentioned above, B-SAFE it is particularly applicable to beam-like structures (notwithstanding its capability of dealing with arbitrary structures), thus illustrations are typically shown for such structures. An example is shown in Figure 2-a, depicting a finite element (FE) model of a thin-walled beam (TWB) structure with elliptic cross-section and subject to fixed-free boundary conditions, where the $x, y, z$ axes correspond to the positive edgewise, flapwise and spanwise directions, respectively. In this case, the geometry allows to generate a structured mesh for which the finite elements are aligned with radial (i.e. axial) and perimetral orthogonal lines, a convenient feature for constructing the B-SAFE model although unstructured meshes can also be handled. Figure 2-b shows a cross-section of the model structure, here called a slice, the perimetral length of which is normalized to unity by defining an arbitrary initial point at which the arc-length position is $\varsigma=0$, proceeding in a counter-clockwise direction and ending at the same initial point at which $\varsigma=1$.

\subsection{Methodology}

The construction of a B-SAFE model starts with the extraction of modal eigenvector data from a parent finite element model representing the structure under study. In a second phase the modal eigenvector data are reorganized into three types of groups. A group of the first type contains the eigendata for each type of nodal direction (or Degree-of-Freedom, DOF) $d$ retained in the analysis, where $1 \leq d \leq N_{d}$. For the purpose of the B-SAFE model construction, and depending on the type of finite element used, each node can contain up to 6 translational $(x, y, z)$ and rotational $\left(\theta_{x}, \theta_{y}, \theta_{z}\right)$ directions. In practice, only translational directions $x, y, z$ are retained, as nodal rotations can be later inferred based on the deformed configuration, thus $N_{d}=3$. A group of the second type contains the eigendata for each mode $m$ retained in the analysis $\left(1 \leq m \leq N_{m}\right)$, where only a few modes $N_{m}$ are sufficient for representing the linear structural behaviour within a range of interest. A group of the third type contains the eigendata for each cross-section (or slice $s$ ) retained in the analysis $\left(1 \leq s \leq N_{s}\right)$. For a structured 
FE mesh, a slice can be defined as a path along the perimeter crossing all the FE nodes located at a fixed radial coordinate, and in this case is equivalent to a cross-sectional path.

The definition of a slice can be visualized in Figure 3, where several views of a generic slice are shown. Figure 3 -a shows the representative model in a deformed configuration corresponding to the $3^{\text {rd }}$-mode displacement profile, with a cross-sectional cut at $5 \%$ of the beam's radial length (i.e. $z=0.05$, as measured from the root). The resulting perimetral path, or slice, is illustrated in Figure 3-b as the arrowed path crossing all the FE-nodes contained at that fixed radial position. Given that the FE mesh is perfectly structured, the slice's plane is orthogonal to the beam's main axis, therefore equivalent to a cross-section. A front view of this slice is depicted in Figure 3-c for both its undeformed and deformed geometry, the latter corresponding to the $3^{\text {rd }}$-mode displacement profile. The undeformed slice's perimetral path is normalized to unity, represented by $\varsigma$ and running from zero to one in a counterclockwise direction. Although a slice is fully equivalent to a cross-section in the latter example, the more general term slice is preferred as it can also handle non-structured meshes by defining irregular perimetral paths having small-to-moderate radial excursions and interpolating the data to a selected fixed radial coordinate. To obtain an accurate B-SAFE representation, the number of slices $\left(N_{s}\right)$ along the radial direction should equate the number of finite elements in the radial direction.

The organization of the eigendata within the three groups described above can be visualized in the left column of Figure 4 for the exemplified slice/mode pair ( $z=0.05$ and $3^{\text {rd }}$-mode), separated into edgewise, flapwise and spanwise directions, respectively. In each case, the slice's perimetral path $\checkmark$ has been unfolded to allow representation on a linear axis and normalized to unity, while the vertical axis $\phi$ measures the magnitude of the eigenvector's value at each FE node contained in the slice. The figures in the right column represent corresponding data for all the slices contained in the representative model, where the radial coordinate has been also normalized to unity, running from zero at the root to unity at the tip. Because each slice's perimetral length is normalized to unity independently of the length of other slices, even a structure with variable cross-section will be mapped to a rectangular plane similar to those shown in the latter figures. For a complete B-SAFE description, eigenvector data for all other remaining modes need to be characterized in a similar fashion.

With the eigendata reorganized within the aforementioned groups, B-SAFE can be constructed as described next. First, eigenvector data for each slice/direction/mode $(s, d, m)$ triplet is fitted to a single so-called perimetral B-spline $P_{s d m}(\varsigma)$ :

$$
P_{s d m}(\varsigma)=\sum_{a=1}^{N_{Q}} \psi_{a, \kappa}(\varsigma) \boldsymbol{Q}_{a}(\varsigma, \phi)
$$


where $0 \leq \varsigma \leq 1$ represents an arbitrary perimetral position within the unfolded and normalized slice's perimetral length, the range of the sub-indexes $s, d, m$ are $1 \leq s \leq N_{s}, 1 \leq d \leq N_{d}, 1 \leq m \leq N_{m}$, and each perimetral function is characterized by $N_{Q}$ control points $\boldsymbol{Q}_{a}(\varsigma, \phi)$.

Three examples of perimetral B-splines are shown in Figure 5, fitting the data previously shown in the left column of Figure 4, here characterized by 18, 23, and 14 control points for the edgewise, flapwise and spanwise B-splines, respectively, and with goodnesses of fit above 0.9996, 0.9999 and 0.9996, respectively. The process described is repeated for all slices/directions/modes retained in the analysis, leading to a set of $N_{s} \times N_{d} \times N_{m}$ perimetral B-splines. In what follows, it is assumed that all perimetral B-splines share an identical (but otherwise arbitrary) knot-vector, thereby also sharing the same number of control points $N_{Q}$ located at the same arc-length positions $\zeta$ along the normalized slice's perimeter, a convenient assumption for further fitting processes. Given the latter, and assuming eigendata for a given $d, m$ pair to vary smoothly between consecutive slices (if these are sufficiently close), it is plausible to assume that corresponding control points $Q_{a}$ will also exhibit a smooth transition across consecutive perimetral B-splines, as exemplified next.

For the illustrative model, the validity of this assumption is inspected in Figure 6, displaying the edgewise $/ 3^{\text {rd }}$-mode perimetral B-splines and their associated control points for a sequence of slices located at every $150 \mathrm{~mm}$ from the root to the tip, i.e., leaving out intermediate slices to avoid a cluttered view. As mentioned above and shown in Figure 5-a, these perimetral B-splines are each characterized by 18 control points, each always localized at the same normalized arc-length perimetral positions for any slice. As an example, it can be noticed in Figure 6 that the control point \#10 is always located at a perimetral position of $\zeta=0.53$ independently of the slice considered. It can be seen from Figure 6 that the vertical coordinate $\phi$ (i.e. its eigenvector value) exhibits a smooth transition across nearby perimetral B-splines. Thereby, the transition of the $\zeta, \phi$ coordinates of the said control point along the model's radial direction provides a new data set for fitting a so-called radial $B$-spline for the exemplified direction/mode pair (the edgewise $/ 3^{\text {rd }}$-mode pair, for the present case). This is the radial B-spline is represented in Figure 6 by the thick curve fitting the transition of the exemplified perimetral control point, where the square marks represent the control points of this radial B-spline, here called meta control points to differentiate them from the previous perimetral control points.

The conformation of the data sets involved in the fitting of the radial B-splines is described more formally in Table 1 for a general direction/mode $(d, m)$ pair. For each slice and its associated perimetral B-spline, defined in the first and second columns by its sequential slice number $S$ and corresponding radial coordinate $Z$, respectively, the $\zeta, \phi$ coordinates of each control point $Q_{a}\left(a=1 \ldots N_{Q}\right)$ are listed, each sharing the same arc-length coordinate $\varsigma_{a}$ as explained previously. The $\phi$-coordinate of 
each control point $Q_{a}$, expressing the eigenvector's value for the selected ( $d, m$ ) pair, is identified as $\phi_{a, s}$, where $s$ represents the number of slice or perimetral function, ranging between $1 \leq s \leq N_{s}$. The assumption of smoothness for the transition of a given control point $Q_{a}$ implies that the data set $\left\{\varsigma_{a}, \phi_{a, 1}, \varsigma_{a}, \phi_{a, 2}, \ldots, \varsigma_{a}, \phi_{a, N s}\right\}_{d, m}$ is suitable to be fitted by a radial B-spline $R_{a d m}$, and the set of all radial B-splines for the selected $(d, m)$ pair can be expressed as $\bigcup_{a=1}^{N_{Q}} R_{a d m}$.

In this way, a set of $N_{Q} \times N_{d} \times N_{m}$ radial B-splines $R_{a d m}$ can be constructed, each capturing the transition of a given control point $Q_{a}$ across all slices for a given $(d, m)$ pair and characterized by an arbitrary number of radial control points $N_{W}$ and an arbitrary knot-vector, as they are not subject to further fitting. Each radial B-spline has the following form:

$$
R_{a d m}(r)=\sum_{b=1}^{N_{W}} \psi_{b, \kappa}(r) W_{b}(r, \phi)
$$

where sub-index $b$ represents the control point $W_{b}$ to be retrieved by the radial B-spline, $0 \leq r \leq 1$ is an arbitrary normalized radial position, $\psi_{b, \kappa}(r)$ are the radial basis functions, and $W_{b}(r, \phi)$ are $N_{W}$ control points $\left(b=1 \ldots N_{W}\right)$ lying in the $r, \phi$ plane, where $\phi$ is an axis orthogonal to $r$ representing the range of values of the perimetral control $Q_{a}$. The radial B-spline shown in Figure 6 is illustrated in more detail in Figure 7, characterized by 14 meta control points.

The radial B-splines include functions of functions, that is, nested functionals capable of retrieving, upon simple evaluation, eigenvector data at arbitrary coordinates $x, y, z$ not restricted to $\mathrm{FE}$ nodes. For example, the eigenvector $\varphi_{n m}$ corresponding to Degree-of-Freedom (DOF) $n$, mode $m$ and localized at slice $s$ can be retrieved as follows:

$$
\varphi_{n m} \approx P_{s d m}\left(\bigcup_{a=1}^{N_{Q}} R_{a d m}(r), \varsigma_{n}\right)=\Gamma_{s d m}\left(\varsigma_{n}\right)
$$

where $\Gamma_{s d m}\left(\varsigma_{n}\right)$ is a nested functional evaluated at coordinate $\varsigma_{n}=f\left(x_{n}, y_{n}\right)$, which represents the perimetral position along the $s^{\text {th }}$ slice's perimeter corresponding to the spatial $x$-coordinate of DOF $n$ and associated to a direction $d$, and the slice $s$ is understood to be located at a radial coordinate $z_{n}$. Physical displacements $w(t)$ can be recovered from classical modal analysis [25], which for DOF $n$ is: 


$$
w_{n}(t)=\sum_{m=1}^{N_{m}} \varphi_{n m} q_{m}(t) \approx \sum_{m=1}^{N_{m}} \Gamma_{s d m}\left(\varsigma_{n}\right) q_{m}(t)=\sum_{m=1}^{N_{m}} \Theta_{s d m}\left(\varsigma_{n}, t\right)
$$

where $q_{m}(t)$ are scalar modal displacements absorbed by functionals $\Theta_{s d m}\left(\varsigma_{n}, t\right)$, the latter yielding a modal contribution to the physical displacement at DOF $n$ and time $t$. It is worth noticing that the functionals $\Gamma_{s d m}\left(S_{n}\right)$ are load-independent, thus an arbitrary change in load requires only recalculating $q_{m}(t)$ to get the new functionals $\Theta_{s d m}\left(\varsigma_{n}, t\right)$, a core feature which provides the means for using the same B-SAFE model within iterative dynamic analyses. This completes the description of the BSAFE method.

\subsection{B-SAFE vs. Poly-SAFE}

The advantage of using B-splines instead of canonical polynomials (as in Poly-SAFE) for fitting perimetral eigenvalues can be illustrated in Figure 8 , containing edgewise $/ 3^{\text {rd }}$-mode eigendata for the slice localized at $z=0.05$ (data already shown in Figure 4-a). In Figure 8-a, a single canonical polynomial of increasingly higher order $(10,20,30$ and 40$)$ is fitted to this data. It can be seen that the fits in the first three cases are rather unsatisfactory as evidenced by the low goodness-of-fit values of $0.8228,0.993$ and 0.9928 , respectively. The $30^{\text {th }}-40^{\text {th }}$-order functions, although the latter exhibiting a reasonable goodness of fit just above 0.996 , were unable to yield usable results due to the high powers involved, systematically leading to over-flow. This situation can be relieved by partitioning the data set into two halves, each covering half of the slice's perimeter. Then, a goodness of fit of $>0.9995$ can be achieved (see Figure $8-b$ ) with a polynomial degree as low as $n=5$. However, this workaround requires manual user intervention which may turn into a heavy pre-processing burden in the case of complicated modal patterns.

\subsection{Accuracy of B-SAFE}

The accuracy of the B-SAFE model can be assessed by comparing its results against those obtained with its parent FE model (the benchmark). Given the large amount of data to be compared, suitable metrics have to be defined to perform simple yet objective comparisons. In a beam-like structure, there are mainly two structural behaviours of interest: the first is the radial deflection or "beam-behaviour", representing the overall displacement of the structure without regard to the local deformations experienced at the cross-sections. In many cases, this overall behaviour is sufficient for analyses dealing with solid or thick-walled cross-sections, or when the primary objective is to satisfy geometrical constraints. Most reduced-order approaches for dealing with slender structures, such as wind turbine blades, aim at reproducing this beam-like behaviour, e.g. through conventional 1D modal analysis [28]. The second behaviour of interest is the local deformation profile of the cross-sections, rarely available in reduced-order models but essential for a detailed calculation of the stress field at the shell material. For critical applications or thin-walled structures, the importance of the latter cannot be overemphasised, as it represents the starting point for a trustworthy Progressive Failure Analyses (PFA, see [3]). In most structures, the magnitudes of the radial deflection and the cross-section deformation profile can differ by several orders, thus different measurement tools are needed to assess the accuracy of each. 
For each direction (i.e. edgewise, flapwise and spanwise), the displacement field at each slice can be decoupled into two components: an average or mean value and a variation around this mean. The first represents the overall displacement of the slice as a whole, that is, the structure's beam-behaviour or radial deflection profile, while the second represents the local deformation profile of the cross-section. A schematic example of this decoupling is illustrated in Figure 9, showing the complete (9-a) and decoupled (9-b) flapwise displacements of a certain slice, where the mean signal is subtracted from the original so the decoupled components are centred at zero. The accuracy of the mean component at each slice can be estimated by the B-SAFE's deviation from the FEM's counterpart, where two normalization procedures are proposed to avoid artificial large errors when comparing two near-zero values: the first (type-I) normalizes the mean's error at each slice with respect to the largest displacement yielded by the FE model at the analysed direction (edgewise, flapwise or spanwise):

$$
\text { Type-I Normalized Error }(\%)=\left|\frac{\left(\bar{x}_{B-S A F E}-\bar{x}_{F E M}\right) / \bar{x}_{F E M}}{\left|x_{F E M}\right|_{\max d i r}} \times 100\right|_{S}
$$

where $\bar{x}$ is the mean value of the displacement field at the analysed slice, $\left|x_{F E M}\right|_{\max \text { dir }}$ stands for the maximum displacement yielded by the FE model at the analysed direction and the sub-index $S$ indicates that the error measures applies to the slice $s$ under analysis. The second normalization (type-II) is similar but normalizes the error with respect to the largest displacement yielded by the FE model for any direction, thereby always yielding a smaller measure:

$$
\text { Type-II Normalized Error }(\%)=\left|\frac{\left(\bar{x}_{B-S A F E}-\bar{x}_{F E M}\right) / \bar{x}_{F E M}}{\left|x_{F E M}\right|_{\max }} \times 100\right|_{S}
$$

The accuracy of the second component, the variation around the mean, can be estimated by the goodness of fit of the B-SAFE field with respect to the benchmark's field.

\section{Results and discussion of a case study}

The case study consists of an ANSYS finite-element model of a $9.2 \mathrm{~m}$ composite wind turbine blade reported in [24], where the reader can find the full set of details of its geometry and material layup. For the sake of completeness, a brief description is included here. The model's geometry is shown in Figure 10 without the top shell and consists in a variable cross-section across four radial segments, each spanning the radial length shown in Table 2. The material layup (i.e. thickness, material and fibreorientation) at the top and bottom shells, described in Tables 3 and 4, is uniform within each radial segment. An internal web runs from a radial coordinate of $1 \mathrm{~m}$ (as measured from the root) up to a radial coordinate of $8.2 \mathrm{~m}$, with material layup as shown in Table 5. The ANSYS FE model consists of 93,100 eight-node multi-layer shell-281 elements, 280,000 nodes and 1,680,000 DOF.

Two separate load cases were applied to the FE model, simulating the distributed loads and torques that the action of the wind would induce on the blade, albeit in a simplified manner. The first load case is represented in Figure 11-a by two positive flapwise loads of $500 \mathrm{~N} / \mathrm{m}$ acting at the web's top and 
bottom points, respectively, both distributed along the web's length. In addition, a positive couple of 50 $\mathrm{N} / \mathrm{m}$ was applied at the endpoints of the cross-section and distributed along the same length as the flapwise load. The second load case is shown in Figure 11-b, represented by two positive edgewise loads of $500 \mathrm{~N} / \mathrm{m}$ acting at the web's top and bottom points, respectively, both distributed along the web's length.

Three B-SAFE models based on cubic B-splines were generated, each retaining the first 5, 10 and 20 modes yielded by the ANSYS FE model and each producing a semi-analytical functional representing the structure's displacement field. The structure of the B-SAFE database is shown in Table 6, containing the number of control points needed to characterize the edgewise, flapwise and spanwise displacement fields for each mode up to the $20^{\text {th }}$ mode. As an example, fitting the $3^{\text {rd }}$-mode required a total of 1,295 control points, out of which the spanwise, flapwise and edgewise fields required 408,440 and 447 control points, respectively. The 5-, 10- and 20-mode B-SAFE models were each characterized by a total of $6,650,13,365$ and 28,482 control points, respectively. To assess the accuracy of each model versus their parent FE model, three measurement schemes were applied: a) a superimposed visual comparison of the overall radial deflection or "beam-behaviour", as represented by the mean value of the displacement field at all slices, b) the type-I and type-II normalized errors of the same mean, measuring the accuracy by which B-SAFE can recover the radial deflection profile at each slice, and c) the goodness of fit of the slice's deformation profile, representing the ability of B-SAFE to reproduce the crosssection's deformation. It is noticed that these three measurements were separately applied for the edgewise, flapwise and spanwise displacements.

For the first load case, the left column of Figure 12 contains the results for the edgewise, flapwise and spanwise radial deflections, respectively, where the labels $5 \mathrm{M}, 10 \mathrm{M}$ and $20 \mathrm{M}$ stand for the 5-, 10- and 20-mode B-SAFE models, respectively. Corresponding results for the second load case are shown in right column of the same figure. Type-I and type-II normalized errors are shown in Figure 13, whereas the coefficients of determination of the cross-sections' deformation profiles are illustrated in Figure 14, both figures following a similar arrangement as in Figure 12.

As far as the radial deflection is concerned, it is conspicuous from Figure 12 that B-SAFE and the benchmark (i.e. the original FE model) yield virtually undistinguishable results in all analysed cases, with type-I and type-II normalized errors below $2 \%$ and $0.8 \%$, respectively. In general for both load cases, the largest errors occurred for the $5 \mathrm{M}$ model given its inability to reproduce complex deformation patterns, particularly at a radial coordinate of around 1-2 $\mathrm{m}$ where a sudden enlargement of the cross-section occurs, also at a radial coordinate of $7.2 \mathrm{~m}$ where the shell's thickness experiences a sudden drop from $10 \mathrm{~mm}$ to $5 \mathrm{~mm}$, and at a radial coordinate of $8.2 \mathrm{~m}$ where the web ends; all three features act as strain concentrators and therefore increase the complexity of the deformation profile. In general, the error in the radial deflection was reduced by a factor of 3 to 6 by refining to the $10 \mathrm{M}$ and $20 \mathrm{M}$ models, with little difference between the latter two. The relatively higher type-I errors found in the spanwise displacements can be attributed to the high axial stiffness of beam-like structures like the present one, leading to very small displacement values in the spanwise direction. The comparison of two very small values make little sense for the purpose of assessing the accuracy of the displacement field, which is more objectively captured by the type-II error by normalizing the error measurements with respect to the largest displacement obtained. Having said that, and in view of overall low type-I and type-Il errors, it can be concluded that all three models considered (5M,10M, and $20 \mathrm{M}$ ) are adequate for B-SAFE modelling if the primary objective of the analysis is to recreate the radial profile or "beam-behaviour" of the structure. Stated otherwise, the inclusion of five vibration modes allows to fully capture the deflection profiles for the load cases considered. 
In spite of its quite satisfactory performance in terms of modelling the beam-like behaviour, the $5 \mathrm{M}$ model performs poorly when attempting to predict the benchmark's deformation profile of the crosssections. This is shown in Figure 14 where the coefficients of determination $\left(R^{2}\right)$ for the B-SAFE fit to the cross-sectional deformation profile of the parent FE model have been plotted as a function of the radial coordinate of the blade. It can be seen that the R2 values are close to zero at regions of high strains such as those found at the three radial coordinates mentioned above, which have a more significant impact in the cross-section's deformation that in the radial deflection. Upgrading to the 10M and 20M models brings a radical improvement in this measurement, with values around 0.8-1 for the first load case and around 0.97-1 for the second load case, with lowest peaks at the same three precise locations. The lower determination coefficients observed at the first load case can be explained by a relatively weaker geometrical stiffness of the cross-section, thus generating more complex deformation profiles. This is alleviated by upgrading from the $10 \mathrm{M}$ to the $20 \mathrm{M}$ model, raising the coefficient of determination to values around 0.9-1, where the same upgrading produces no significant improvement in the second load case in view of already high values. In conclusion, it can be said that the poor performance of the $5 \mathrm{M}$ model and the significant gain in accuracy experienced by upgrading to the $10 \mathrm{M}$ and $20 \mathrm{M}$ models, is strong evidence that the number and type of modes retained in the construction of the B-SAFE is of paramount importance for capturing the cross-section's deformation profile, especially for thin-walled structures. It would be arguable that even 20 modes are rather few in view of the modest goodnesses of fit observed at some regions. Nonetheless, if an accurate prediction of failure zones is the main output of the analysis, as it is the case for design optimization cycles, attention must be paid to the root and mid-radial positions, where the goodness of fit is sufficiently high for this type of analysis. Otherwise, retaining more modes will always be the safest path although at the expense of added computational effort.

\section{Summary and conclusions}

This paper has introduced the B-SAFE, acronym for "B-spline-based Semi-Analytical Finite Element", extending the capabilities and functionality of the original Poly-SAFE method developed by these authors in [2] by replacing canonical polynomials with B-splines. The paper focuses on the non-trivial modifications and advantages generated by such replacement, where qualitative results drawn from a representative model were used to illustrate the written description. A case study, represented by a finite element model of a $9.2 \mathrm{~m}$ composite wind turbine blade reported in [23], was used for constructing three increasingly refined B-SAFE models, comparing their results with those yielded directly by the parent FE model. The main improvements can be expressed as follows:

- Similar to the original Poly-SAFE, B-SAFE is able to reduce a general FE model to a semi-analytical functional by recursively fitting B-splines to modal data extracted from its parent FE model. These semi-analytical functions are virtually load-independent and can be constructed for any field variable such as displacement, strain, stress, etc., which can be retrieved upon simple evaluation of the functional at selected coordinates not restricted to FE nodes. The local-support feature of B-splines allows a single function of globally low-order (i.e. cubic) to handle highcurvature regions simply by selecting more feature points (knots) in the vicinity of those regions, thus representing a far more advanced fitting technology as compared to power-based polynomials. Although this argument is well-known and true for most kind of data, an important finding in the present work is that the transition of each perimetral control point along the model's radial direction is smooth (for a given direction/mode pair) and can maintain a constant perimetral coordinate if the same knot-vector is applied to all perimetral B-splines. These 
conditions allow each perimetral control point to be accurately fitted to radial B-splines, obtaining nested functionals with highly-condensed information of the FE model.

- The handling of complex geometries previously required either very high-order canonical polynomials to be fitted, making them prone to fitting and numerical instabilities or simply unable to retrieve data upon evaluation, or partitioning the data into smooth portions which could be fitted individually by lower-order functions, thus requiring heavy user-intervention. By using B-splines, a single low-order function (i.e. cubic) could provide a goodness of fit sufficiently high for most engineering purposes, each B-spline characterized by few control points. It can be concluded that the advantages of B-SAFE versus Poly-SAFE are not related to the computational efficiency involved in building the model, but rather to the accuracy, robustness and automation achieved during construction and usage (i.e. evaluation). Given that the B-SAFE is typically built only once prior to iterative calculations, the latter issues have far more impact in a realistic analysis.

- The various numerical experiments performed in the case study indicate that all the B-SAFE models were capable of reproducing the radial deflection, or overall "beam-behaviour", of the structure, with error measures between $0.8 \%$ and $2 \%$. However, the deformation profile of the cross-sections was accurately captured only by the highest-order model (20 modes), producing goodnesses of fit very close to 1 although with peaks as low as 0.9 at specific regions of high strain gradients. This deformation profile is rarely available in reduced-order models and represents essential input data for more advanced analyses (i.e. failure criteria and damage tracking).

- Unlike most numerical models (i.e. FE), which accuracy can be assessed only after the first results have been obtained, the ability of measuring the quality of the B-SAFE model prior to its usage within iterative calculations is an advantageous feature.

Further on, the advanced arithmetic/differential manipulation techniques already available for B-splines open the door for non-trivial extensions of the capabilities and functionality with respect to the original Poly-SAFE. Avenues of future work include the derivation of similar semi-analytical functions for stress fields and specialized failure criteria, for which failure points can be straightforwardly identified without seeking in the entire FE mesh, thus making feasible a near real-time Progressive Failure Analysis (PFA) of large models. For the case of local events modifying the FE model (i.e. damage, nonlinearities), updating specific spatial regions of the B-SAFE without reformulating it entirely would also be advantageous, and there are currently available a number of techniques which can be implemented for that purpose. All these avenues will be explored in future papers.

\section{Acknowledgements}

Support from the following Institutions is greatly acknowledged: Tecnológico de Monterrey, through the Research Chair for Wind Energy, contract No. CAT158; CONACYT, through the Grant SEP-CONACYT CB2009-01-127942. The National Science Foundation under Grant No. 1031036, and the New York State Energy Research and Development Authority (NYSERDA) under Grant Nos. 18812 and 18460. 


\section{References}

\begin{tabular}{|c|c|}
\hline [1] & tttp://www.comsol.com/company/ \\
\hline$[2]$ & ttp://www.ansys.com/About+ANSYS/Contacts+and+Locations \\
\hline [3] & tp://www.ascgenoa.com/contact/index.jsp \\
\hline [4] & 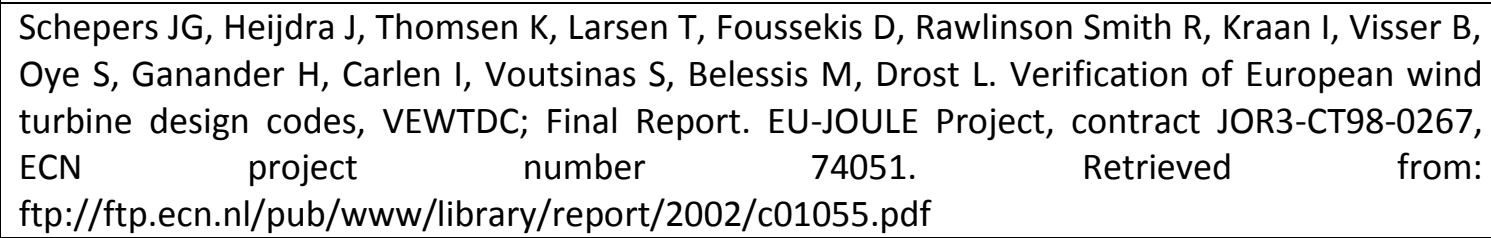 \\
\hline [5] & $\begin{array}{l}\text { Hansena MOL, Sørensena JN, Voutsinas S, Sørensenc N, Madsen HA. State of the art in wind } \\
\text { turbine aerodynamics and aeroelasticity. Prog Aerosp Sci 2006; 42(4): 285-330. }\end{array}$ \\
\hline [6] & $\begin{array}{l}\text { Heijdra JJ. The accuracy of wind turbine design codes derived from VEWTDC results. ECN- } \\
\text { WIND Note-03-6071; } 2003 \text {. }\end{array}$ \\
\hline [7] & $\begin{array}{l}\text { Petersen T. The aeroelastic code HawC -model and comparisons. ETDEWEB ID: 283718, } \\
\text { September 01; 1996. <http://hawc2proeve.wordpress.com/hawc2-newest-version/ } \\
\text { http://orbit.dtu.dk/fedora/objects/orbit:79840/datastreams/file_7703110/content> }\end{array}$ \\
\hline [8] & -cell anisotr \\
\hline [9] & 13-439. \\
\hline [10] & eoryc \\
\hline [11] & Gjelsvik \\
\hline [12] & $\begin{array}{l}\text { sov VZ, Leontev UN. Beams, plates and shells on elastic foundations. Israel Program for } \\
\text { entific Translations; } 1966 .\end{array}$ \\
\hline [13] & $\begin{array}{l}\text { Garg DP, Zirky MZ, Anderson GL. Current and potential future research activities in adaptive } \\
\text { structures: and ARO perspective. Smart Mater Struct 2001: 10(4): 610-623. http:// } \\
\text { dx.doi.org/10.1088/0964-1726/10/4/304. }\end{array}$ \\
\hline [14] & $\begin{array}{l}\text { rescu L, Song O. Thin-walled composite beams: theory and application. Springer; } 2006 . \\
\text { N-10 1-4020-3457-1. }\end{array}$ \\
\hline [15] & $\begin{array}{l}\text { dges DH. Nonlinear composite beam theory. Progress in astronautics and aeronautics. } \\
\text { erican Institute of Aeronautics and Astronautics; } 2006 \text { [eISBN: 978-1-60086-682-1, print } \\
\text { N: 978-1-56347-697-6, doi: } 10.2514 / 4.866821] \text {. }\end{array}$ \\
\hline [16] & $\begin{array}{l}\text { Chandra R, Stemple AD, Chop } \\
\text { extensional loads. J Aircr } 199\end{array}$ \\
\hline [17] & $\begin{array}{l}\begin{array}{l}\text { Barbero EJ, Lopez-Anido R, Davalos JF. On the mechanics of thin walled laminated composite } \\
\text { beams. J. Compos Mater }\end{array} \text { J. 1993; } \\
\text { http.//dx.doi.org.10.1177/002199839302700804. }\end{array}$ \\
\hline [18] & $\begin{array}{l}\text { C. Zhang, S.Wang, , Structure mechanical modelling of thin-walled closed-section composite } \\
\text { beams, Part 1: Single cell cross section, Composite Structures, Volume 113, July 2014, Pages } \\
\text { 12-22, ISSN 0263-8223, http://dx.doi.org/10.01016/j.compstruct.2014.03.005. }\end{array}$ \\
\hline [19] & $\begin{array}{l}\text { Cárdenas D., Elizalde H., Marzocca P., Probst O., Ramirez R., Toledo J.P. The Poly-SAFE } \\
\text { method: A semi-analytical representation of finite element models via nested polynomial } \\
\text { reduction of modal data. Composite Structures } 2014 ; 111: 301-316 \text {. }\end{array}$ \\
\hline$[20]$ & $\begin{array}{l}\text { Farin G. Curves and Surfaces for CADG: a practical guide. San Francisco: Morgan Kaufmann } \\
\text { Publishers Inc, } 2002 \text {. }\end{array}$ \\
\hline [21] & Piegl L., Tiller W. The NURBS book. New York: Springer-Verlag, 1995. \\
\hline$[22]$ & Piegl L. Til \\
\hline [23] & Park H., Lee J-H. B-spline curve fitting based on adaptive curve refinement using domir \\
\hline
\end{tabular}




\begin{tabular}{|l|l|}
\hline & points. Computer-Aided Design 2007; 39(6): 439-451. \\
\hline$[24]$ & $\begin{array}{l}\text { Cárdenas D., Escárpita A.A., Elizalde H., Aguirre J.J., Ahuett H., Marzocca P., Probst O. } \\
\text { Numerical Validation of a finite element thin-walled beam model of a composite wind turbine } \\
\text { blade. Wind Energy 2012; 15(2): 203-223. }\end{array}$ \\
\hline$[25]$ & $\begin{array}{l}\text { Erwins DJ. Modal testing, theory, practive and application (mechanical engineering research } \\
\text { studies: engineering dynamics series). Research studies Pre; 2 } \\
\text { September } 26,2000 .\end{array}$ \\
\hline$[26]$ & $\begin{array}{l}\text { Prautzsch H., Boehm W., Paluszny M. Bézier and B-spline Techniques. New York: Springer- } \\
\text { Verlag, 2002. }\end{array}$ \\
\hline$[27]$ & \begin{tabular}{l} 
Bingham N. H., Fry J.M. Regression: Linear Models in Statistics. London: Springer-Verlag, 2010. \\
\hline$[28]$
\end{tabular} \\
$\begin{array}{l}\text { Tony Burton, Nick Jenkins, David Sharpe, Ervin Bossanyi. Wind Energy Handbook, 2nd Edition, } \\
\text { ISBN: 978-0-470-69975-1, June 2011 }\end{array}$ \\
\hline
\end{tabular}


TABLES

Computationally efficient reduction of modal data from finite element models by nested sets of B-splines

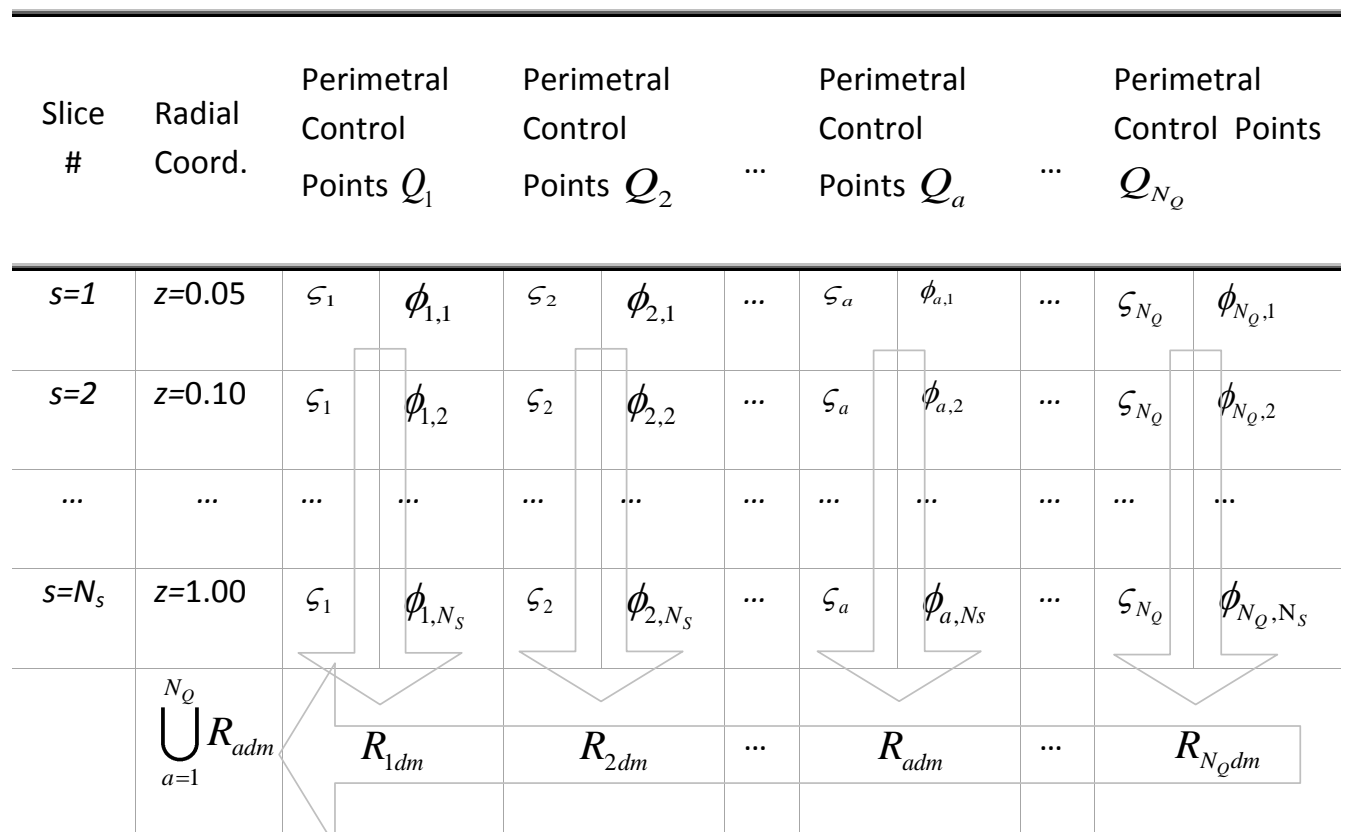

Table 1. Conformation of the data sets involved in the fitting of the radial B-splines for a general direction/mode $(d, m)$ pair. 


\begin{tabular}{ccc}
\hline Radial segment & $\begin{array}{l}\text { Initial radial } \\
\text { coordinate }(\mathrm{m})\end{array}$ & $\begin{array}{l}\text { Final radial } \\
\text { coordinate }(\mathrm{m})\end{array}$ \\
\hline L1 & 0 & 1 \\
L2 & 1 & 3.2 \\
L3 & 3.2 & 7.2 \\
L4 & 7.2 & 9.2 \\
Web & 1 & 8.2 \\
\hline
\end{tabular}

Table 2. Length of radial segments and web.

\begin{tabular}{|c|c|c|c|c|c|}
\hline \multicolumn{4}{|c|}{ Thickness (mm) } & \multirow[t]{2}{*}{ Material } & \multirow[t]{2}{*}{ Angle $\left({ }^{\circ}\right)$} \\
\hline L1 & L2 & L3 & L4 & & \\
\hline 2.5 & 2 & 1.25 & 0.625 & 2 & 45 \\
\hline 2.5 & 2 & 1.25 & 0.625 & 2 & -45 \\
\hline 10 & 1 & 5 & 2.5 & 1 & 0 \\
\hline 2.5 & 2 & 1.25 & 0.625 & 2 & -45 \\
\hline 2.5 & 2 & 1.25 & 0.625 & 2 & 45 \\
\hline
\end{tabular}

\begin{tabular}{ccccc}
\hline Material & $1 /(\mathrm{C} 520)$ & $2 /(\mathrm{C} 260)$ & $3 /(3 / 4$ Mat $)$ & $4 /$ (Balsa) \\
\hline E11 (MPa) & 48200 & 43000 & 7580 & 2070 \\
E22 (MPa) & 11700 & 8900 & 7580 & 2070 \\
G12 (MPa) & 6480 & 4500 & 6480 & 140 \\
V12 & 0.30 & 0.27 & 0.30 & 0.22 \\
$\rho\left(\mathrm{g} / \mathrm{cm}^{3}\right)$ & 1.87 & 1.87 & 1.67 & 0.14 \\
\hline
\end{tabular}

Table 4. Mechanical properties of materials. 


\begin{tabular}{ccc}
\hline Thickness $(\mathrm{mm})$ & Material & Angle $\left(^{\circ}\right)$ \\
\hline 0.186 & 2 & 45 \\
0.186 & 3 & 0 \\
0.186 & 2 & -45 \\
9.525 & 4 & 0 \\
0.186 & 2 & 45 \\
0.186 & 3 & 0 \\
0.186 & 2 & -45 \\
\hline
\end{tabular}

Table 5. Material layup of the web. 


\begin{tabular}{|c|c|c|c|c|c|}
\hline \multirow{2}{*}{ Mode } & \multicolumn{3}{|c|}{$\begin{array}{c}\text { Number of control points by } \\
\text { DOF }\end{array}$} & \multirow[b]{2}{*}{ Sum } & \multirow[b]{2}{*}{$\begin{array}{l}\text { Accumulated } \\
\text { Sum }\end{array}$} \\
\hline & Span & Flap & Edge & & \\
\hline 1 & 422 & 456 & 528 & 1,406 & 1,406 \\
\hline 2 & 387 & 439 & 464 & 1,290 & 2,696 \\
\hline 3 & 408 & 440 & 447 & 1,295 & 3,991 \\
\hline 4 & 429 & 448 & 421 & 1,298 & 5,289 \\
\hline 5 & 407 & 466 & 488 & 1,361 & 6,650 \\
\hline 6 & 459 & 401 & 390 & 1,250 & 7,900 \\
\hline 7 & 456 & 424 & 395 & 1,275 & 9,175 \\
\hline 8 & 463 & 472 & 489 & 1,424 & 10,599 \\
\hline 9 & 453 & 435 & 480 & 1,368 & 11,967 \\
\hline 10 & 427 & 459 & 512 & 1,398 & 13,365 \\
\hline 11 & 485 & 465 & 522 & 1,472 & 14,837 \\
\hline 12 & 473 & 438 & 471 & 1,382 & 16,219 \\
\hline 13 & 510 & 463 & 468 & 1,441 & 17,660 \\
\hline 14 & 499 & 472 & 539 & 1,510 & 19,170 \\
\hline 15 & 522 & 450 & 540 & 1,512 & 20,682 \\
\hline 16 & 549 & 453 & 530 & 1,532 & 22,214 \\
\hline 17 & 561 & 468 & 583 & 1,612 & 23,826 \\
\hline 18 & 508 & 524 & 561 & 1,593 & 25,419 \\
\hline 19 & 592 & 476 & 564 & 1,632 & 27,051 \\
\hline 20 & 439 & 488 & 504 & 1,431 & 28,482 \\
\hline Sum & 9,449 & 9,137 & 9,896 & 28,482 & 28,482 \\
\hline
\end{tabular}

Table 6. Number of control points needed to characterize the displacement fields of the case of study. 


\section{FIGURES}

Computationally efficient reduction of modal data from finite element models by nested sets of B-splines

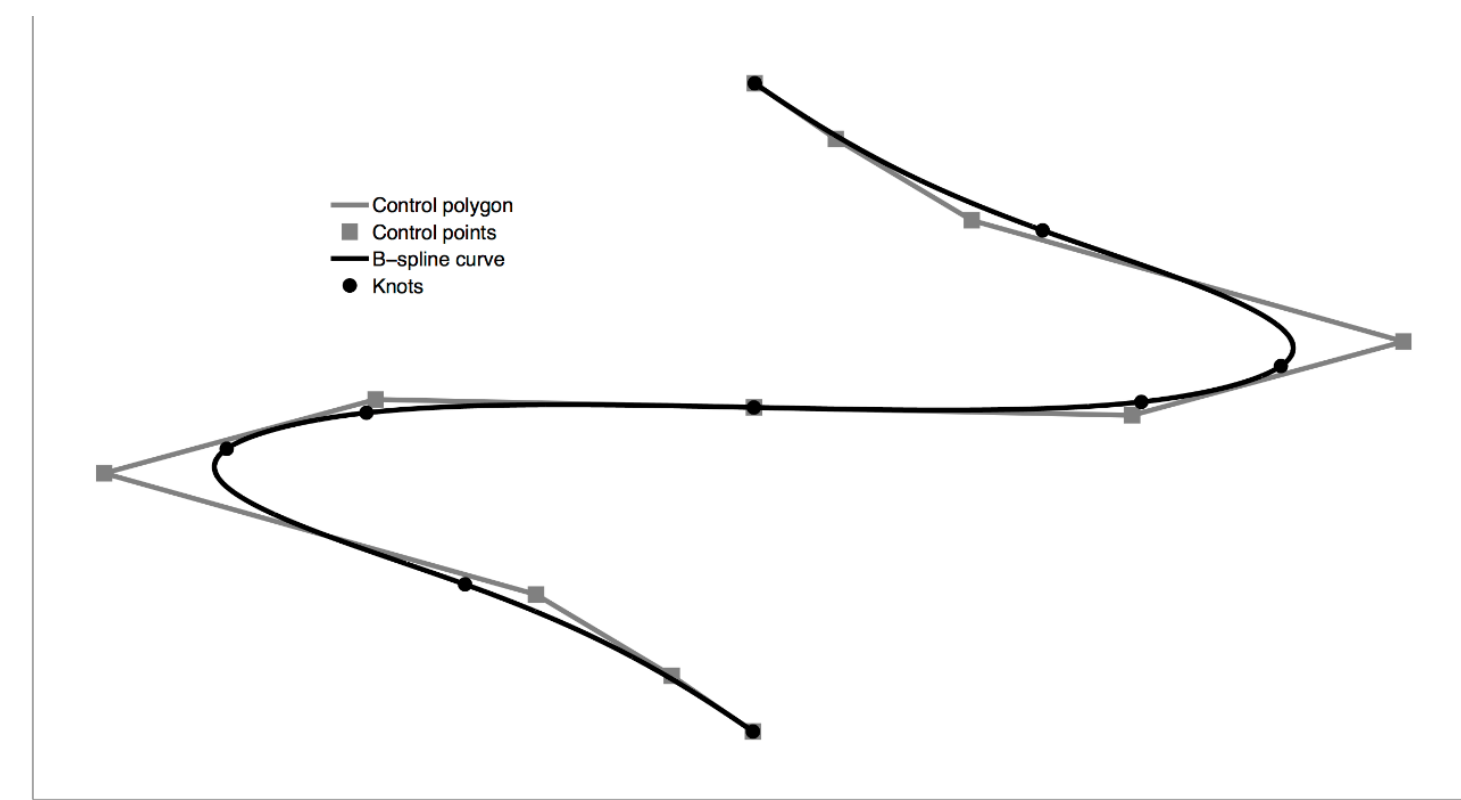

Figure 1. A cubic B-spline curve with the corresponding control polygon and knots. 

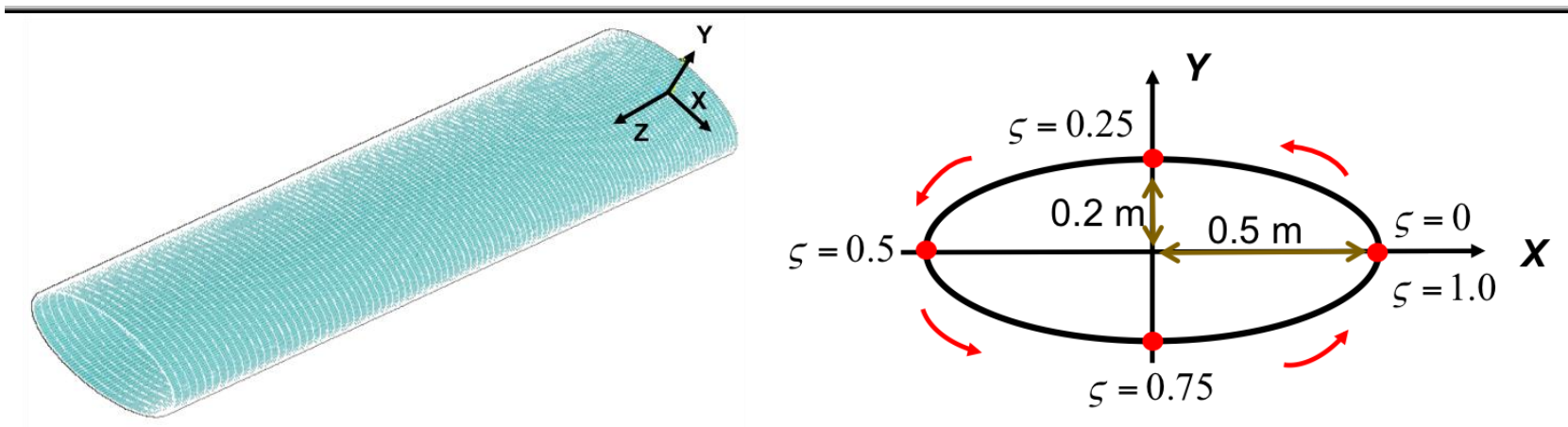

Figure 2. a) Finite element model of a thin-walled beam structure with elliptic cross-section, and b) a generic slice (or cross-section) of the model, which normalized perimeter starts at $\varsigma=0$ at the right end, proceeding in a counter-clockwise direction and ending at $\varsigma=1$ at the same initial point. 


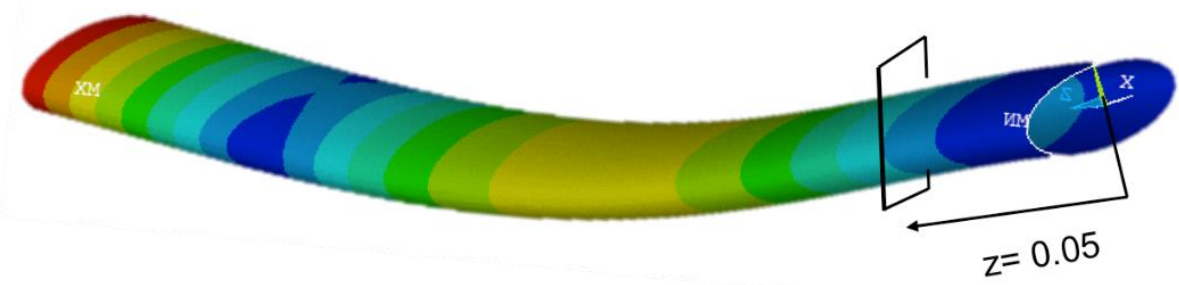

b)

c)
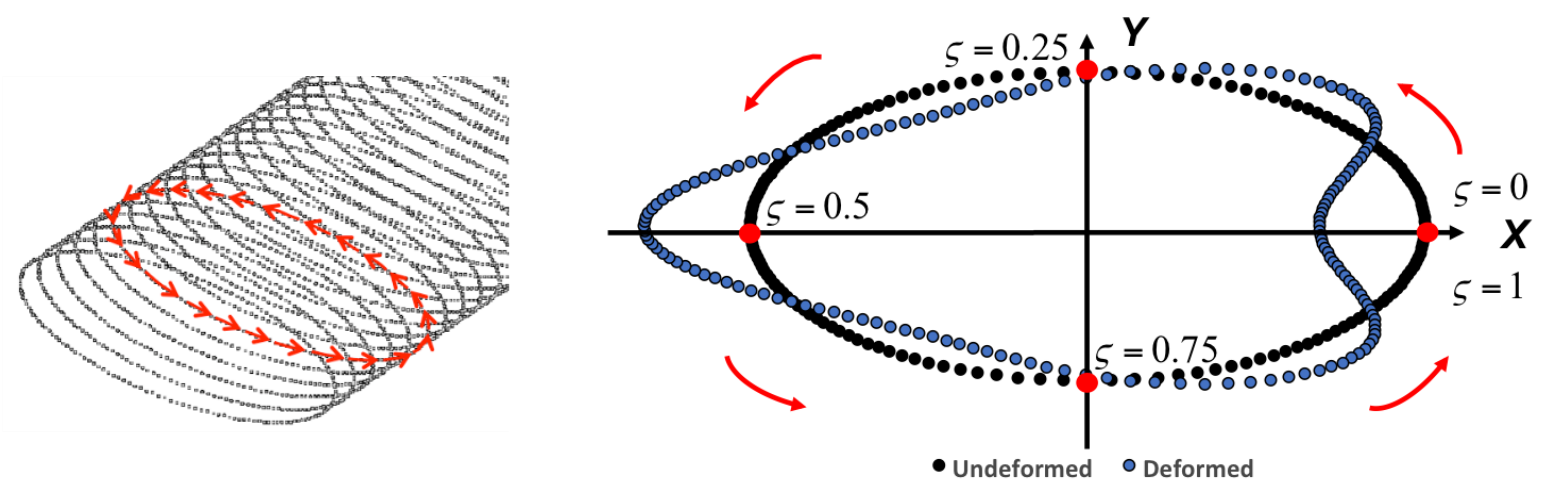

Figure 3. Several views of a generic slice of an illustrative beam-like structure. a) Deformed beam-like structure corresponding to the $3^{\text {rd }}$-mode displacement field and a cross-sectional cut localized at a radial coordinate of $z=0.05, b$ ) the resulting slice represented by the arrowed path crossing the finite element nodes of the parent model (illustrated by dot marks) at that radial position, and c) front view of the slice in its undeformed and deformed configuration, the latter corresponding again to the $3^{\text {rd }}$-mode displacement profile. 
a)
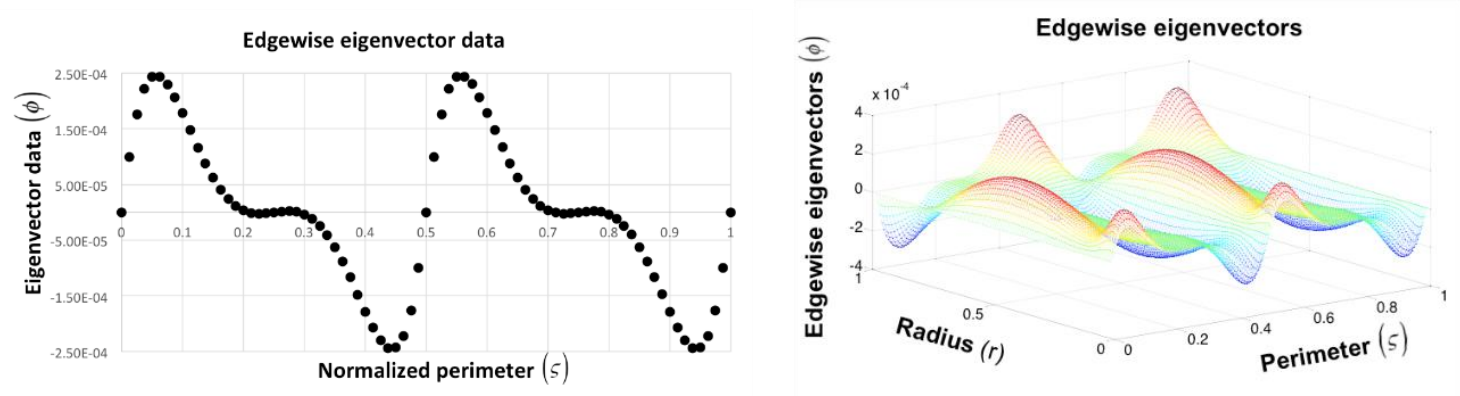

b)
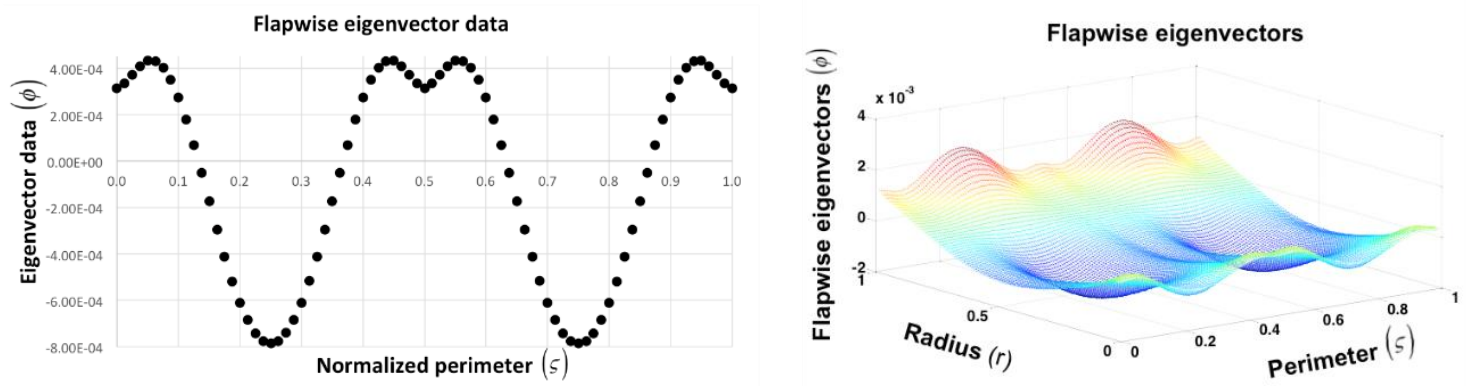

c)
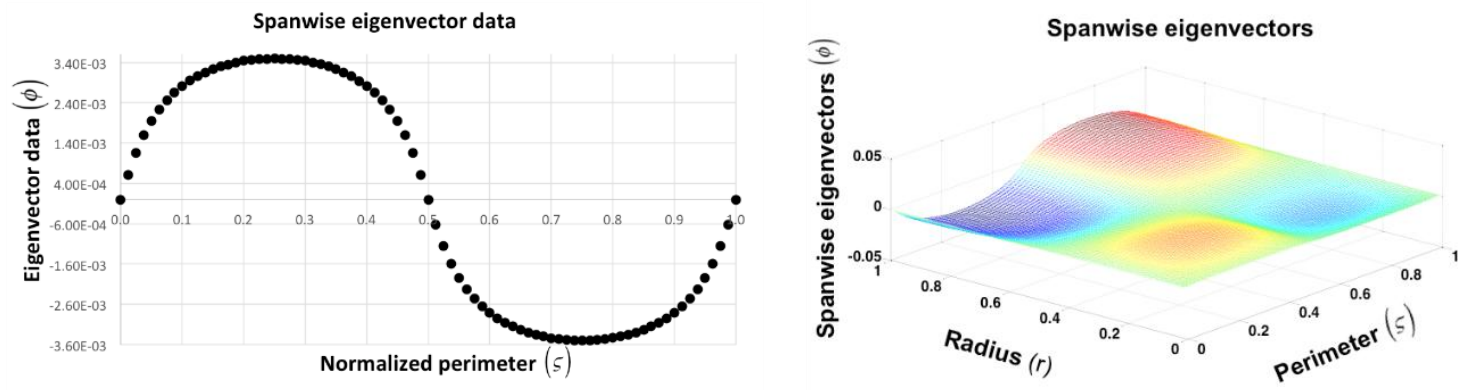

Figure 4. $3^{\text {rd }}$-mode displacement eigenvector data of the illustrative model shown in Figure 2. The figures in the left column show data corresponding to the slice located at $z=0.05$, separated into $a$ ) edgewise, $b$ ) flapwise and c) spanwise directions. The figures in the right column show the eigenvector data for all slices of the illustrative model. In all cases, the radial and perimetral paths have been normalized to unity. 
a)

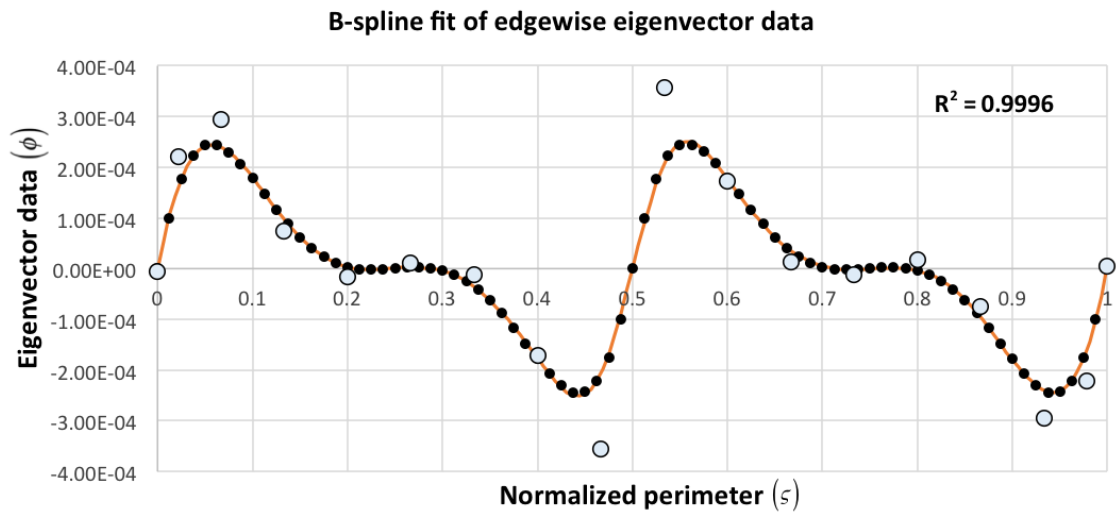

- Eigenvector data - Perimetral B-spline $\bigcirc$ Perimetral control points

B-spline fit of flapwise eigenvector data

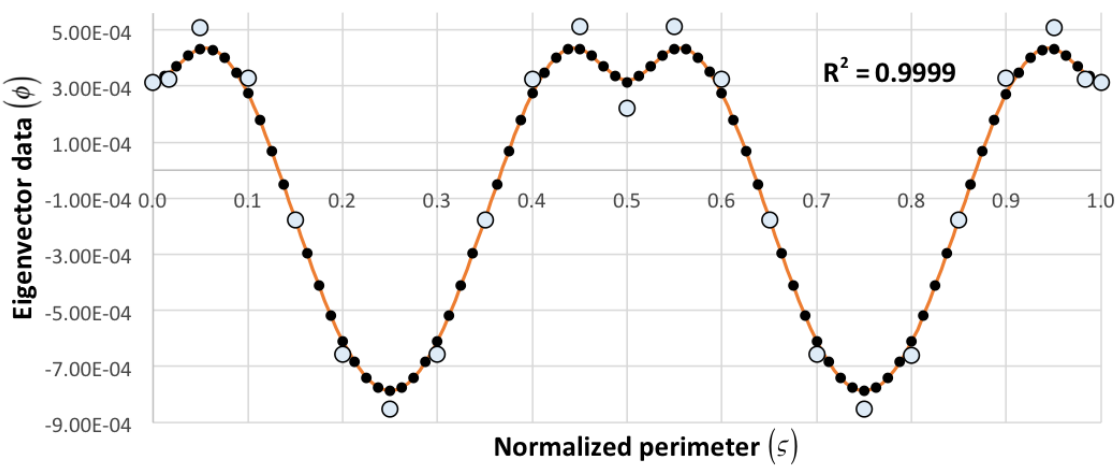

- Eigenvector data - Perimetral B-spline $\bigcirc$ Perimetral control points

b)

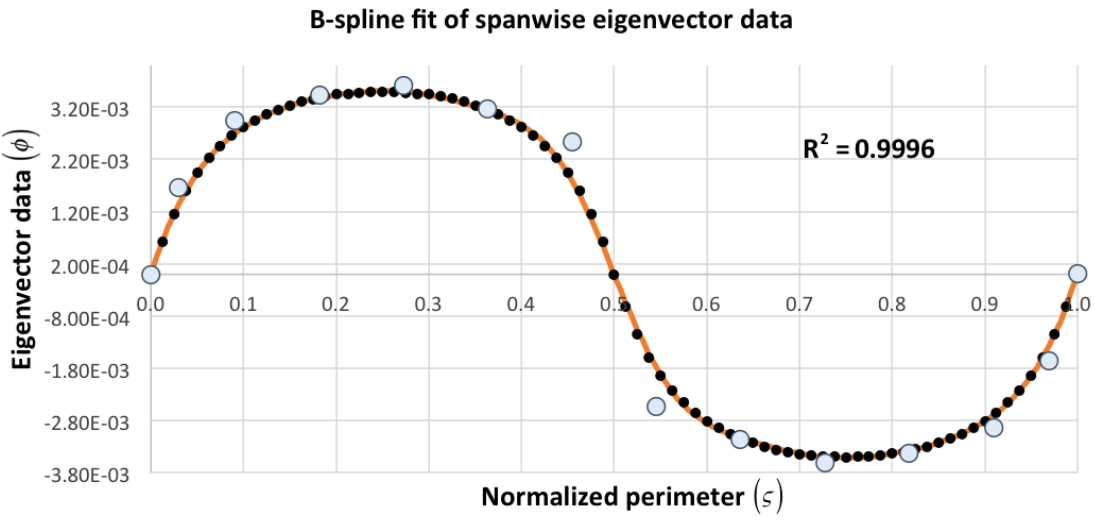

- Eigenvector data - Perimetral B-spline $\bigcirc$ Perimetral control points

Figure 5. Perimetral B-splines fitted to the eigenvector data shown in Figure 4 (left column), corresponding to the pair slice@z=0.05/3rd-mode and separated into a) edgewise, b) flapwise, c) spanwise directions. The functions are characterized by 18, 23 and 14 control points, respectively, with a goodness of fit above 0.9996, 0.9999 and 0.9996, respectively. 

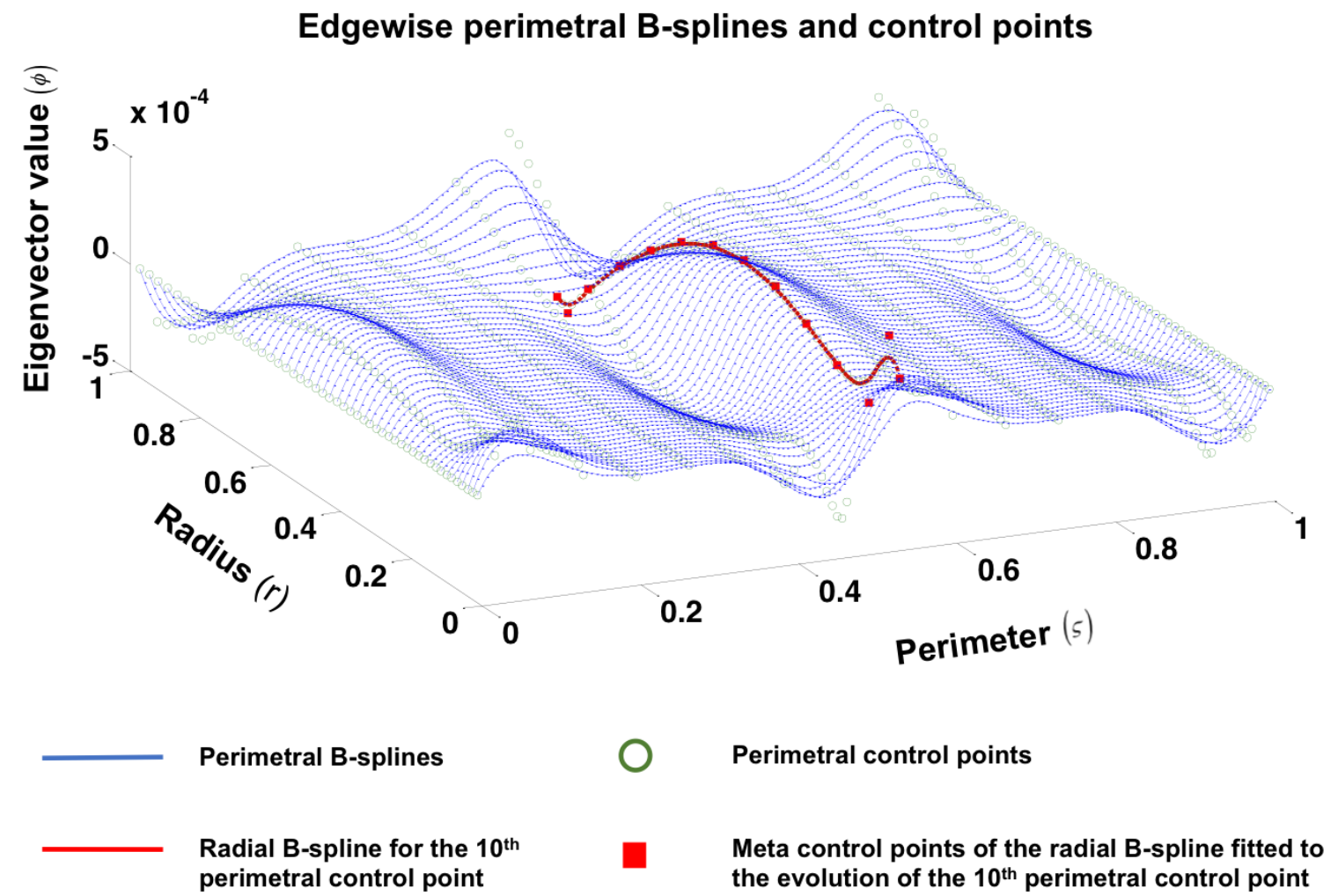

Perimetral control points

Radial B-spline for the $10^{\text {th }}$

Meta control points of the radial B-spline fitted to the evolution of the $10^{\text {th }}$ perimetral control point

Figure 6. Perimetral B-splines and associated control points for the edgewise $/ 3^{\text {rd }}$-mode pair. Also shown in thick-line is the radial B-spline and its corresponding meta control points fitting the transition of the $10^{\text {th }}$ perimetral control point. 


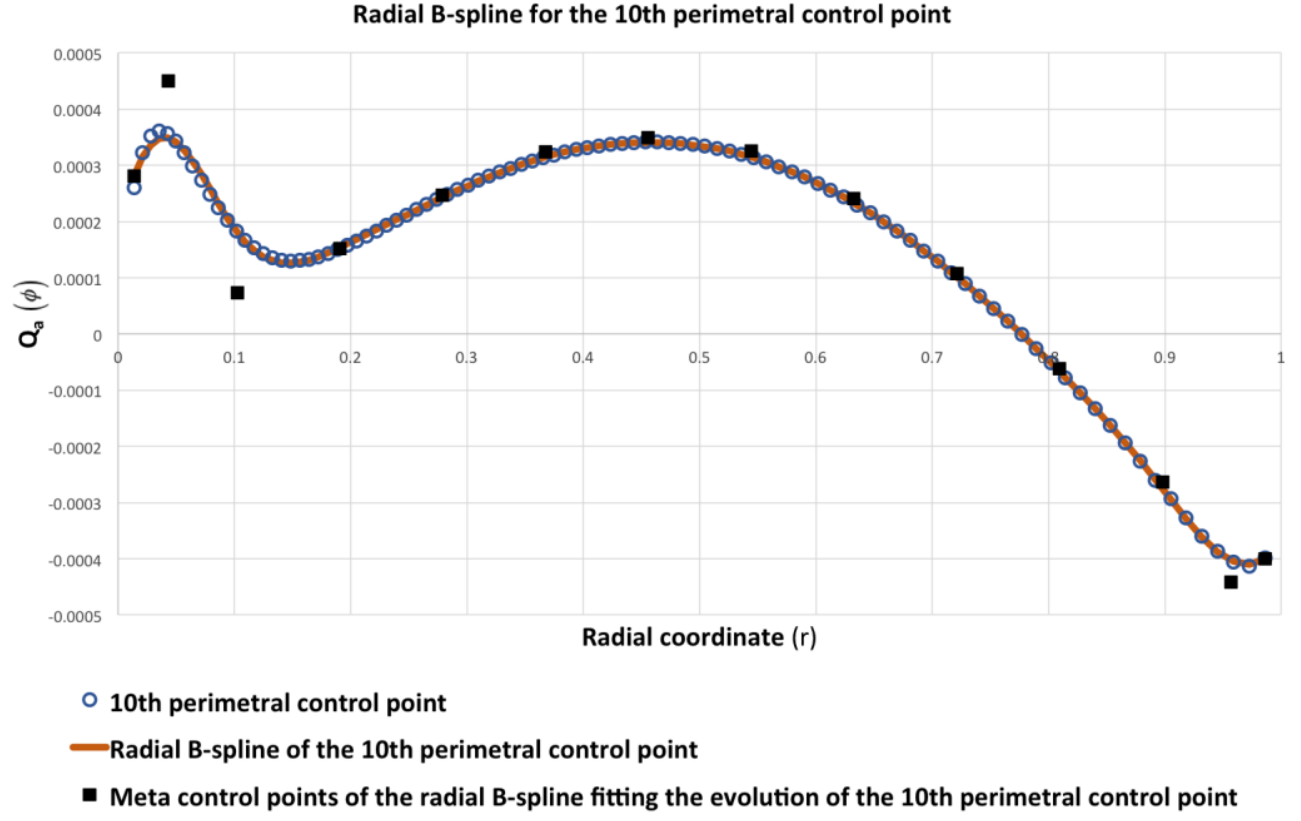

Figure 7. Radial B-spline fitting the transition of the $10^{\text {th }}$ perimetral control point for the edgewise $/ 3^{\text {rd }}$ mode pair. 
Polynomial fit of edgewise eigenvector data

a)

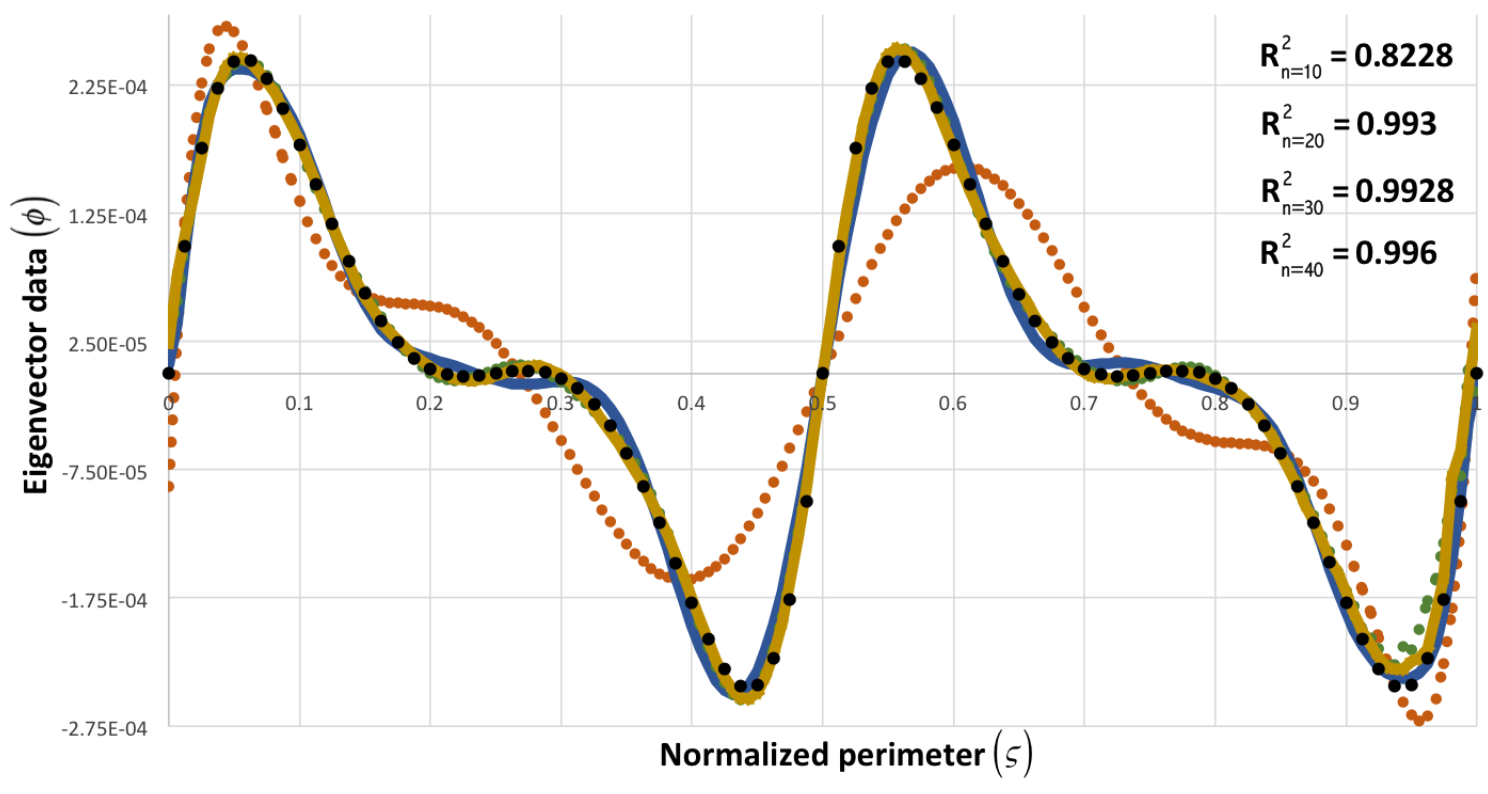

- Eigenvector data $\cdots n=10 \quad n=20 \cdots n=30 \quad n=40$

b)

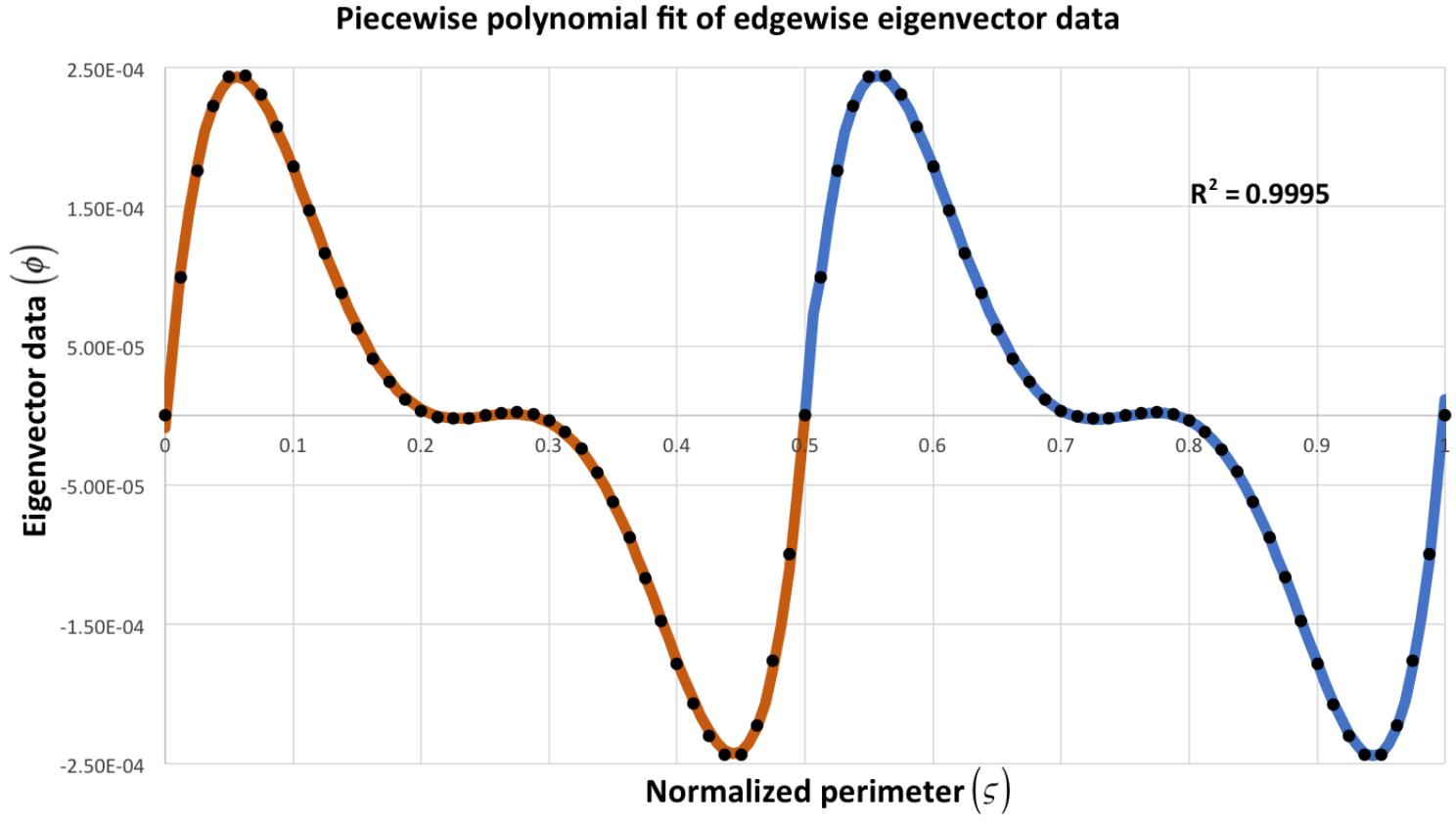

- Eigenvector data ${ }_{n=5} \quad n=5$

Figure 8. Edgewise $/ 3^{\text {rd }}$-mode eigenvalues and fitted canonical polynomials. a) four polynomials of order $10,20,30$ and 40 fitted to the whole $\varsigma$-domain, b) two $5^{\text {th }}$-order polynomials each fitted to a half $\varsigma$ domain. 


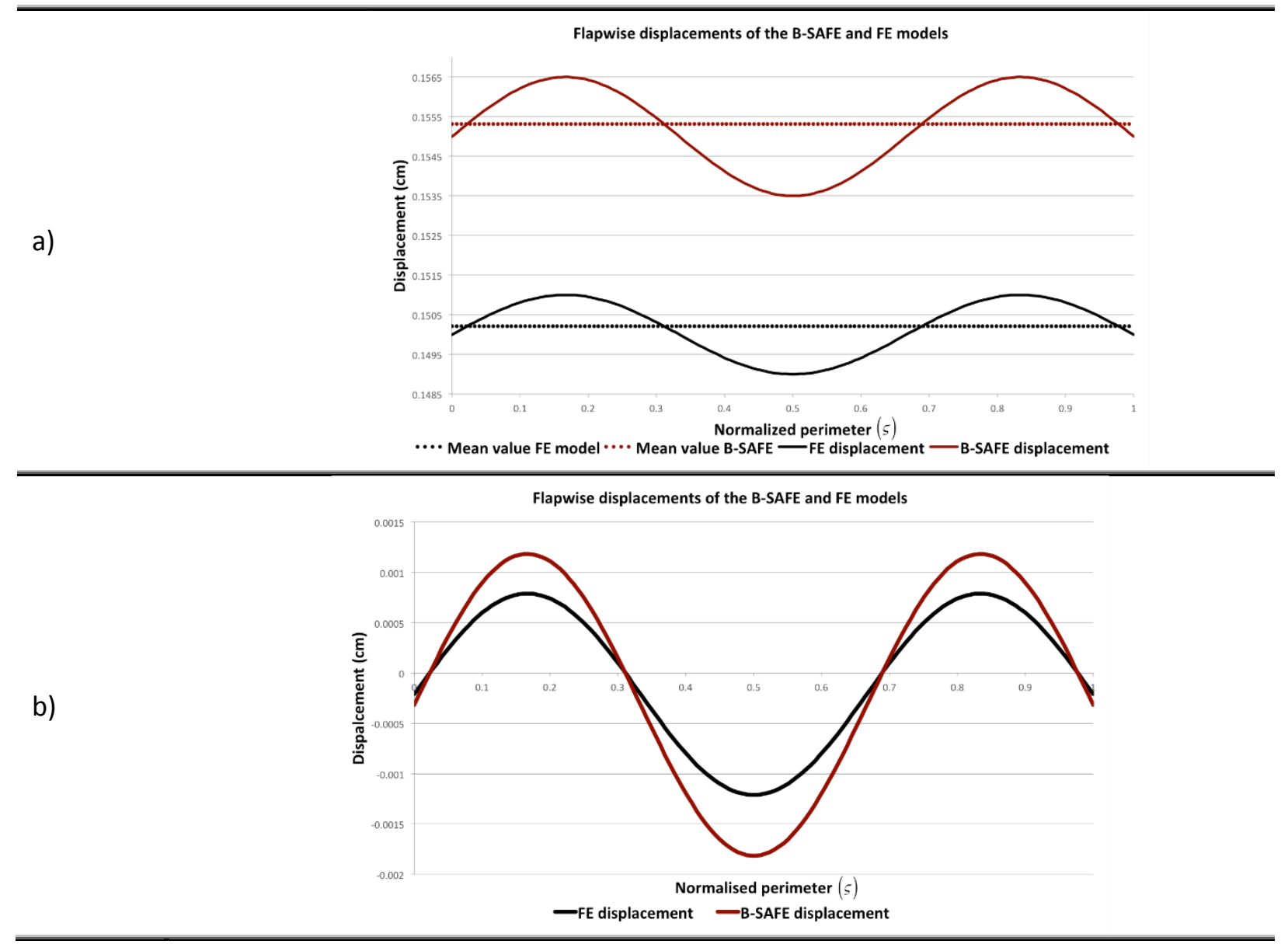

Figure 9. a) Complete and b) decoupled displacements for a schematic flapwise displacement of a certain slice, where the decoupled components are centred on zero by removing the mean component. 


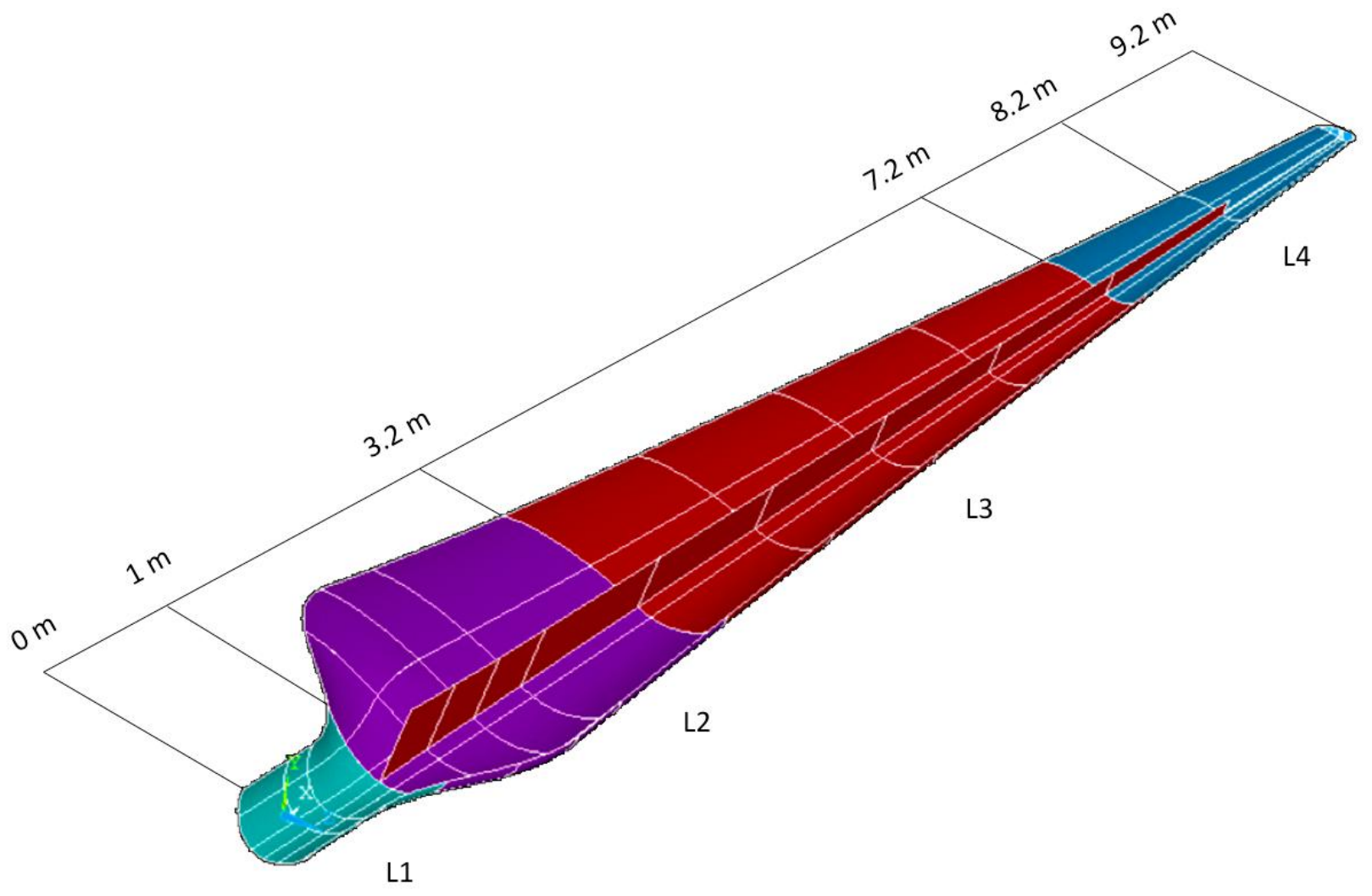

Figure 10 . Geometry of the wind turbine blade model. 


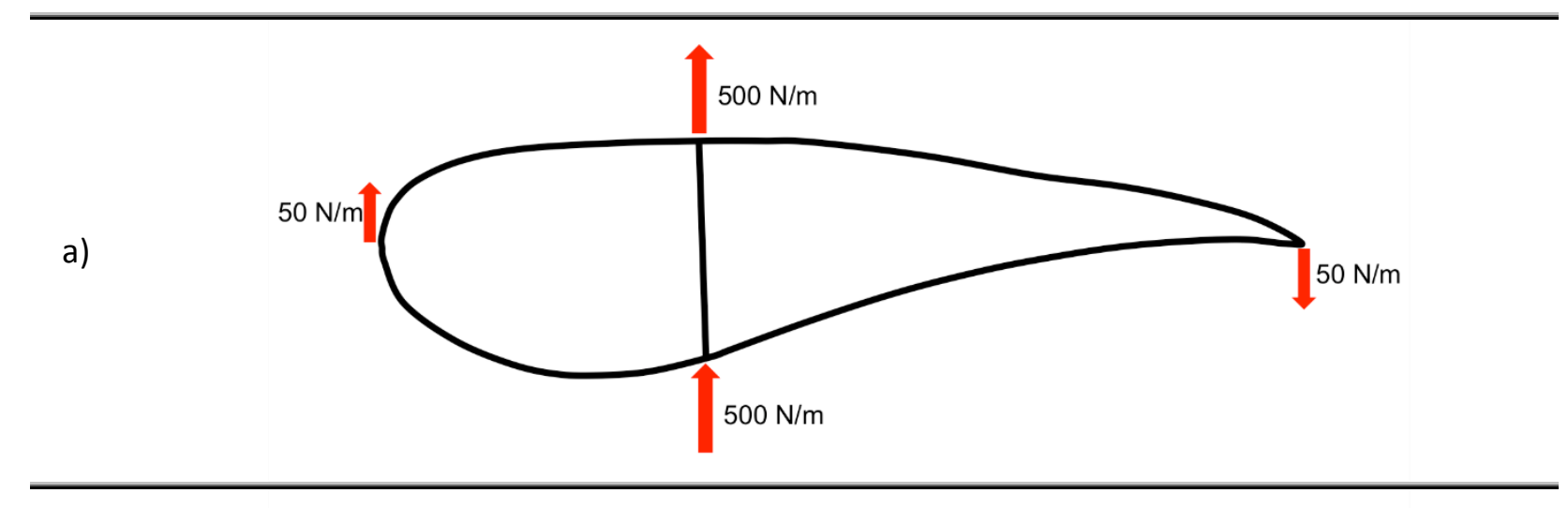

b)

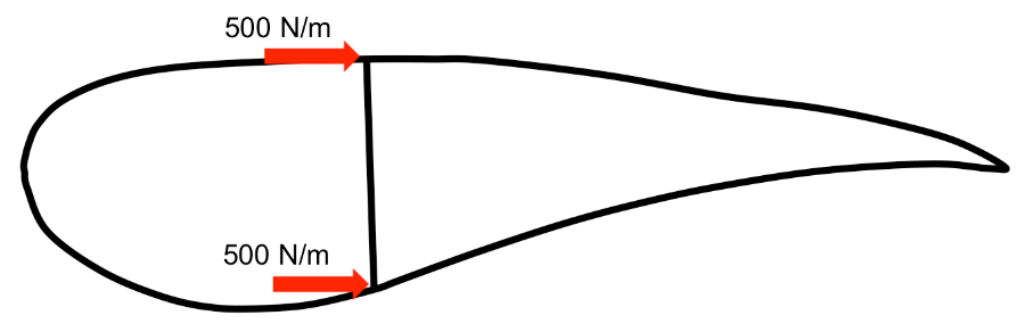

Figure 11. a) First load case and b) second load case for the sample study. 


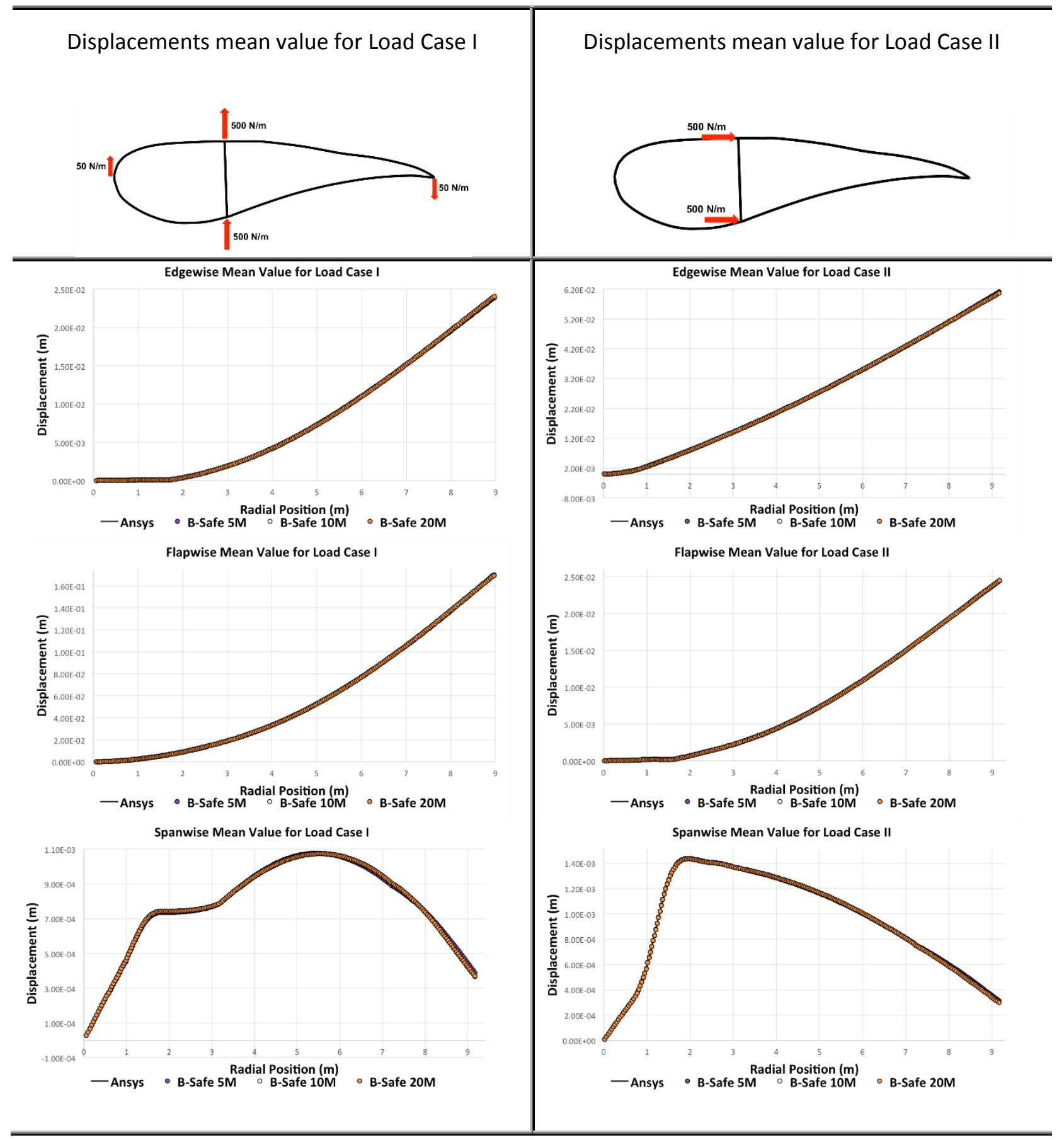

Figure 12. Left column shows the edgewise, flapwise and spanwise displacement fields for the first load case, while the right column shows the corresponding results for the second load case. 


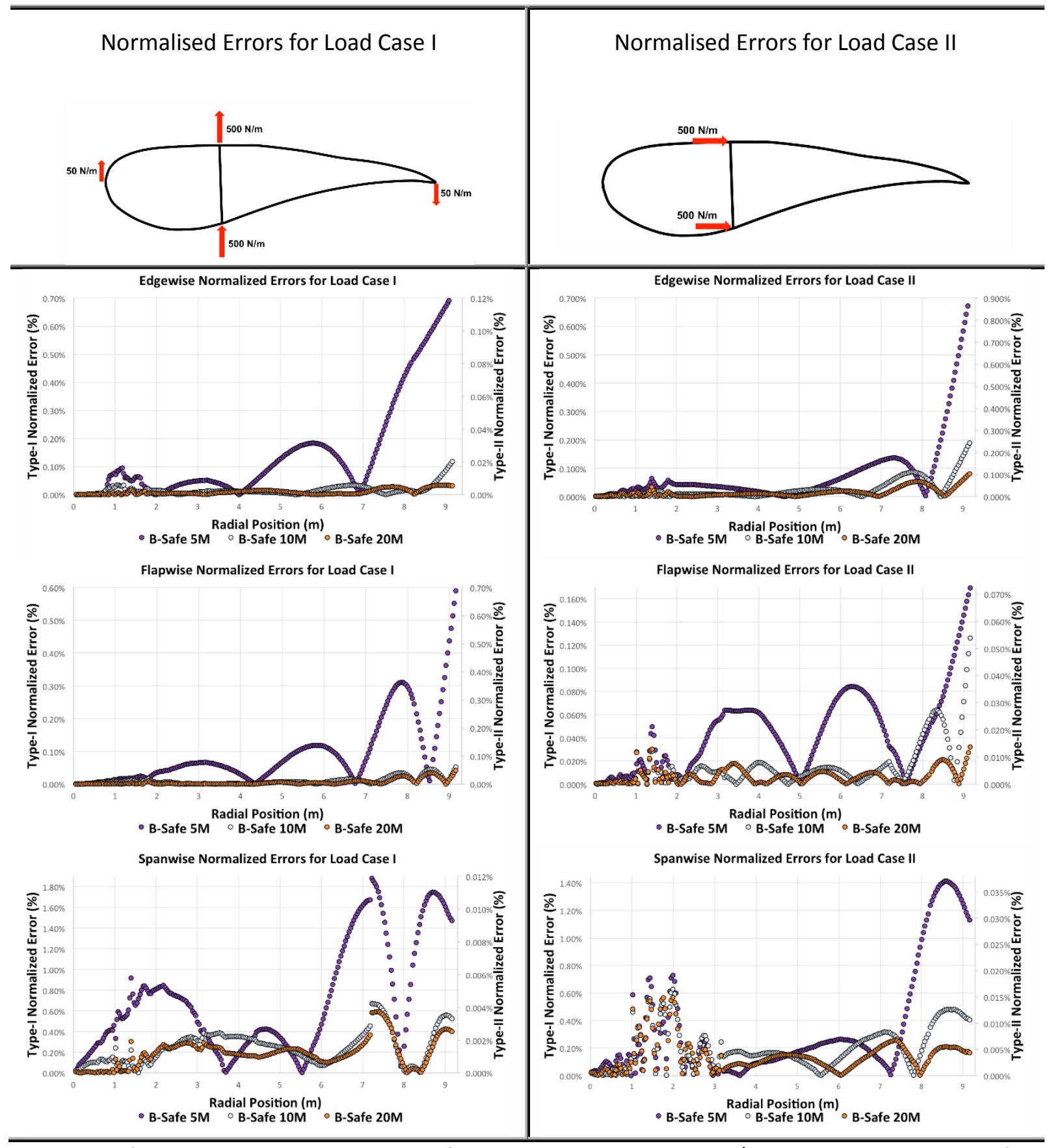

Figure 13. Left column shows the edgewise, flapwise and spanwise type-l/type-II normalised errors for the first load case, while the right column shows the corresponding results for the second load case. 


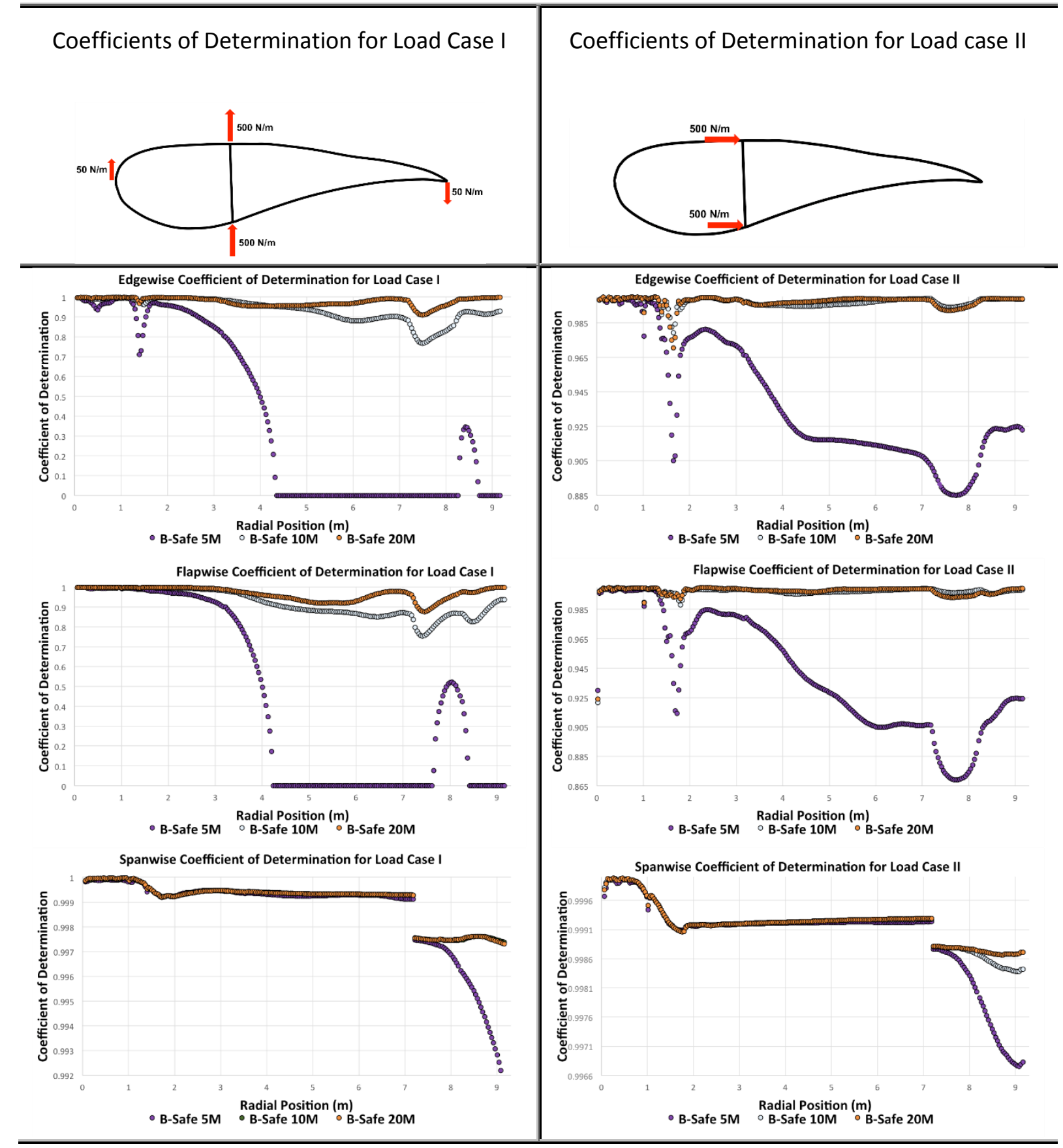

Figure 14. Left column shows the edgewise, flapwise and spanwise coefficient of determinations for the first load case, while the right column shows the corresponding results for the second load case. 